\title{
Layer-dependent magnetic phase diagram in FenGeTe2 ( $3 \leq n \leq 7)$ ultrathin films: coexistence of localized and itinerant electronic states of Fe with variable valence
}

Qinxi Liu

Dalian University of Technology

Jianpei Xing

Dalian University of Technology

Zhou Jiang

Dalian University of Technology

Yu Guo

Dalian University of Technology

Xue Jiang ( $\sim$ jiangx@dlut.edu.cn )

Dalian University of Technology

Yan Qi

Dalian Minzu University

Jijun Zhao

Dalian University of Technology

\section{Article}

Keywords: magnetic, temperature, ultrathin, films, high

Posted Date: October 25th, 2021

DOI: https://doi.org/10.21203/rs.3.rs-961504/v1

License: (c) (1) This work is licensed under a Creative Commons Attribution 4.0 International License. Read Full License

Version of Record: A version of this preprint was published at Communications Physics on June 3rd, 2022. See the published version at https://doi.org/10.1038/s42005-022-00921-3. 


\title{
Layer-dependent magnetic phase diagram in $\mathrm{Fe}_{n} \mathrm{GeTe}_{2}(3 \leq n \leq 7)$ ultrathin films: coexistence of localized and itinerant electronic states of $\mathrm{Fe}$ with variable valence
}

Qinxi Liu ${ }^{1}$, Jianpei Xing ${ }^{1}$, Zhou Jiang ${ }^{1}$, Yu Guo ${ }^{1}$, Xue Jiang ${ }^{1 *}$, Yan Qi ${ }^{2,1 *}$, Jijun Zhao ${ }^{1}$

${ }^{1}$ Key laboratory of Material Modification by Laser, Ion and Electron Beams (Dalian University of Technology), Ministry of Education, Dalian, 116024, China

${ }^{2}$ School of Physics and Materials Engineering, Dalian Minzu University, Dalian 116600, China

\begin{abstract}
Two-dimensional (2D) ferromagnets with high Curie temperature $\left(\mathrm{T}_{\mathrm{C}}\right)$ are highly desirable due to their potential applications in spintronic devices. However, they are rarely obtained in experiments mainly due to the challenge of synthesizing high-quality $2 \mathrm{D}$ crystals, and their $T_{C}$ values are below the room temperature. By first-principles calculations, herein we design a family of stable $2 \mathrm{D} \mathrm{Fe}_{n} \mathrm{GeTe}_{2}(4 \leq n \leq 7)$ ultrathin films that are similar to the reported $\mathrm{Fe}_{3} \mathrm{GeTe}_{2}$, which exhibit coexistence of itinerant and localized magnetism. Among them, 2D $\mathrm{Fe}_{3} \mathrm{GeTe}_{2}$ and $\mathrm{Fe}_{4} \mathrm{GeTe}_{2}$ are ferromagnetic metals with $\mathrm{T}_{\mathrm{C}}$ of $138 \mathrm{~K}$ and $68 \mathrm{~K}$, respectively; 2D $\mathrm{Fe}_{5} \mathrm{GeTe}_{2}, \mathrm{Fe}_{6} \mathrm{GeTe}_{2}$ and $\mathrm{Fe}_{7} \mathrm{GeTe}_{2}$ ultrathin films are Néel's P-types, R-type, R-type ferrimagnetic metals with $\mathrm{T}_{\mathrm{C}}$ of $320 \mathrm{~K}, 450 \mathrm{~K}$ and $570 \mathrm{~K}$, respectively. The thickness induced magnetic phase transition is mainly originated from the competition between itinerant and localized states, which is also correlate well with the content of $\mathrm{Fe}^{3+}$ and $\mathrm{Fe}^{2+}$ ions. A valence/orbital dependent magnetic exchange model is proposed to clarify such interesting thickness and composition effect. Our results not only endow 2D Fe-Ge-Te ultrathin films as promising candidates for spintronics at room temperature, but also propose a universal mechanism to understand the magnetic coupling in complex magnetic systems.
\end{abstract}

*Corresponding authors. E-mail: jiangx@dlut.edu.cn (Xue Jiang), qiyan@dlnu.edu.cn (Yan Qi) 
Since the successful exfoliation of monolayer $\mathrm{CrI}_{3}$ and bilayer $\mathrm{CrGeTe}_{3}$ sheets, the family of two-dimensional (2D) magnetic materials have been extraordinarily growing during the past few years. Right now, the range of 2D magnets covers insulators, semiconductors, half-metals, and metals. Among them, the most concerned ones are ferromagnetic (FM) semiconductor, such as $\mathrm{CrX}_{3}, \mathrm{NiX}_{3}, \mathrm{CrGeTe}_{3}$ and $\mathrm{RuX}_{3}\left(\mathrm{X}=\mathrm{Cl}, \mathrm{Br}\right.$ and I). ${ }^{1,2,3,4,5,6}$ Magnetism in these compounds originates from localized $d$ electrons and the magnetic ordering is usually mediated by superexchange interaction between the magnetic ions through the non-metal atoms. The semiempirical Goodenough-Kanamori-Anderson (GKA) rules provide valuable picture to describe the magnetic interactions in these $2 \mathrm{D}$ compounds. ${ }^{7,8,9}$ According to the GKA rules, the ferromagnetism in $2 \mathrm{D}$ semiconductors is mainly derived from $90^{\circ} d-p-d$ superexchange. In this situation, the occupied orbitals overlap with different orthogonal $p$ orbitals of a ligand. It is thus not surprising that weak ferromagnetism is usually found in those systems. As representatives, the observed Curie temperatures $\left(\mathrm{T}_{\mathrm{C}}\right)$ of the $2 \mathrm{D}$ compounds of $\mathrm{CrI}_{3}$ and $\mathrm{CrGeTe}_{3}$ are $45 \mathrm{~K}$ and $30 \mathrm{~K}$, respectively, which are far below the room temperature. ${ }^{1,4}$

Beyond these ferromagnetic semiconductors, metallic ferromagnet is another important member of $2 \mathrm{D}$ ferromagnet. A prominent advantage of the metallic ferromagnet is that its metallic nature enables the interplay between spin and charge degrees of freedom, which are the main concern in spintronics. ${ }^{10}$ The reported metallic ferromagnets, such as $\mathrm{Cr}_{2} \mathrm{BC}, \mathrm{FeSe}_{2}$,

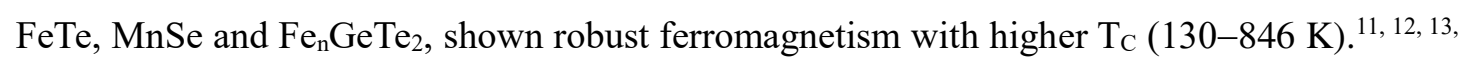
14, 15, 16, 17, 18, 19 Especially, 2D metallic Fe-Ge-Te ternary compounds (FGT) with high $\mathrm{T}_{\mathrm{C}}$ and huge MAE along the $\mathrm{c}$ axis have attracted attention. Among the FGT thin films, $2 \mathrm{D} \mathrm{Fe}_{3} \mathrm{GeTe}_{2}$ was first achieved by cleaving $\mathrm{Fe}_{3} \mathrm{GeTe}_{2}$ bulk crystal onto a gold film evaporated on top of $\mathrm{SiO}_{2} / \mathrm{Si}$ substrate. The polar reflective magnetic circular dichroism measurement confirmed that the $\mathrm{T}_{\mathrm{C}}$ were preserved at $68-130 \mathrm{~K}$ with MAE value of $\sim 2.0 \mathrm{meV}$ at the monolayer limit. ${ }^{13,14}$ Subsequently, Kim et al. have successful synthesized and exfoliated $\mathrm{Fe}_{4} \mathrm{GeTe}_{2}$ flakes and the $\mathrm{T}_{\mathrm{C}}$ for a seven-layer $\mathrm{Fe}_{4} \mathrm{GeTe}_{2}$ system of $7 \mathrm{~nm}$ thickness was determined to be about $270 \mathrm{~K}$. However, the observed MAE reduces from 1.03 to $0.23 \mathrm{~J} / \mathrm{cm}^{3}$ when the composition changes from $\mathrm{Fe}_{3} \mathrm{GeTe}_{2}$ to $\mathrm{Fe}_{4} \mathrm{GeTe}_{2} .{ }^{15}$ Another important member of FGT system is $\mathrm{Fe}_{5-\mathrm{x}} \mathrm{GeTe}_{2}$, May et al. fabricated $\mathrm{FM} \mathrm{Fe}_{5-\mathrm{x}} \mathrm{GeTe}_{2}$ nanoflakes with higher $\mathrm{T}_{\mathrm{C}}$ of $270-310 \mathrm{~K}$ by mechanically exfoliated method. ${ }^{16}$ 
So far, spontaneous magnetization in most of $2 \mathrm{D}$ metallic ferromagnet is generally accepted to be the itinerant electrons, which can be understood by the well-known Stoner model. ${ }^{20}$ The electrons behave ferromagnetic just because of their repulsive Coulomb interaction, while the contributions from lattice and band structure are totally ignored. Beneficial from the delocalized electrons, the reported metallic ferromagnets in most cases shown higher $\mathrm{T}_{\mathrm{C}}$ than ferromagnetic semiconductors. However, lots of works suggested that metallic FGT systems is not conventional Stoner ferromagnets. The itinerant magnetism could not totally explain the variation of $\mathrm{T}_{\mathrm{C}}$ in FGT systems. For example, Dai et al. founded that the increased hydrostatic pressure lead to the enhancement of electron itinerancy but decreased the $\mathrm{T}_{\mathrm{C}}$ in thin $\mathrm{Fe}_{3} \mathrm{GeTe}_{2}$ flakes. ${ }^{21}$ Yang et al. noticed that the band dispersions of $\mathrm{Fe}_{3} \mathrm{GeTe}_{2}$ barely change upon heating towards the ferromagnetic transition near $225 \mathrm{~K}$, which also strongly deviates from itinerant Stoner model. ${ }^{22}$ Deng et al used localized Heisenberg model to properly estimate the magnetic properties in $2 \mathrm{D} \mathrm{Fe}_{3} \mathrm{GeTe}_{2}$, which consistent with the experiment results. ${ }^{14}$ All these results suggest that the local magnetic moments may play a crucial role in the ferromagnetic ordering of Fe-Ge-Te systems. From above experimental reports, we can conclude that 2D metallic FGT are prospective candidates for room-temperature ferromagnet. However, there still exist several unsolved issues, such as the physical origin of their localized magnetism in metallic systems, the validity of Stoner model and Heisenberg model, the effect of composition/thickness, and the influence of magnetic anisotropy.

To reveal these issues, the electronic and magnetic properties of 2D $\mathrm{Fe}_{3} \mathrm{GeTe}_{2}$ were systemically investigated in this work. We found that the five $3 d$ orbitals of $2 \mathrm{D} \mathrm{Fe}_{3} \mathrm{GeTe}_{2}$ could divide into two parts, $\mathrm{a}_{1}\left(d_{\mathrm{z} 2}\right)$ states mostly localized on the Fe sites and give rise to local spin moments, while the other $\mathrm{e}_{1}\left(d_{\mathrm{xy}} / d_{\mathrm{x} 2-\mathrm{y} 2}\right)$ and $\mathrm{e}_{2}\left(d_{\mathrm{xz}} / d_{\mathrm{yz}}\right)$ states are itinerant. According to the orbital occupation behavior of localized $\mathrm{a}_{1}\left(d_{\mathrm{z} 2}\right)$ states and coordination environments, the final valence states for $\mathrm{Fe}$ atoms in $\mathrm{Fe}_{3} \mathrm{GeTe}_{2}$ can been classified into +2 and +3 . For localized spins on Fe atoms, we propose a valence-dependent multipath magnetic coupling mechanism to describe the competition between the interlayer ferromagnetism and antiferromagnetism, while itinerant $\mathrm{e}_{1}$ and $\mathrm{e}_{2}$ states always favor in intra- and interlayer ferromagnetism in $2 \mathrm{D} \mathrm{Fe}_{3} \mathrm{GeTe}_{2}$. Furthermore, the MAE also depended on the valence state of Fe ions, which origin from the coupling between $a_{1}$ and $e_{2}$ states. Based on these findings, we further construct a series of $2 \mathrm{D}$ 
$\mathrm{Fe}_{\mathrm{n}} \mathrm{GeTe}_{2}$ ultrathin films $(4 \leq n \leq 7)$ with different $\mathrm{Fe}$ contents and thicknesses. Their combined effect on magnetic moment, magnetic exchange parameters, and MAE has been discussed. We found the value of $\mathrm{T}_{\mathrm{C}}$ in $\mathrm{Fe}_{\mathrm{n}} \mathrm{GeTe}_{2}$ ultrathin films is indeed depended on the competition between the localization and itinerant magnetism. An interesting thickness-induced magnetic phase transformation from ferromagnets to Néel's P-types ferrimagnets, and then to R-type ferrimagnets are observed in $2 \mathrm{D} \mathrm{Fe}_{\mathrm{n}} \mathrm{GeTe}_{2}$ films, and their $\mathrm{T}_{\mathrm{C}}$ are in the range of $68-570 \mathrm{~K}$. Our results not only reveal a new way to design 2D intrinsic magnets with high Curie temperature but also provide a universal theoretical model for analyzing the itinerant and localized magnetism in complex materials.

\section{Results}

Co-existence of localized and itinerant magnetism in $2 \mathrm{D} \mathrm{Fe}_{3} \mathrm{GeTe}_{2}$. The atomic configuration of $2 \mathrm{D} \mathrm{Fe}_{3} \mathrm{GeTe}_{2}$ is shown in Fig. 1a. Each $\mathrm{Fe}_{3} \mathrm{GeTe}_{2}$ unit has a thickness of five atomic layers. Clearly, there are two types of $\mathrm{Fe}$ atoms with different coordination environments, i.e., trivalent iron $\left(\mathrm{Fe}^{3+}\right)$ and divalent iron $\left(\mathrm{Fe}^{2+}\right)$. The middle of $2 \mathrm{D} \mathrm{Fe} \mathrm{GeTe}_{2}$ is a $\mathrm{Fe}^{2+} \mathrm{Ge}$ layer, sandwiched by bottom and upper $\mathrm{Fe}^{3+}$ layers. The entire surface of each $\mathrm{Fe}^{3+}$ layer is then covered by the atomic layer of Te. The corresponding ratio of number of $\mathrm{Fe}^{3+}$ and $\mathrm{Fe}^{2+}$ is $2: 1$. The $2 \mathrm{D} \mathrm{Fe}_{3} \mathrm{GeTe}_{2}$ is metallic, ${ }^{13}$ as seen from both electronic band structure and total density of states (TDOS) in Supplementary Fig. 1. Non-magnetic (NM), FM, and antiferromagnetic (AFM) states are all considered to determine their ground spin configurations. The corresponding FM and various AFM configurations are shown in Supplementary Fig. 2. Our results indicate that $2 \mathrm{D} \mathrm{Fe}_{3} \mathrm{GeTe}_{2}$ has $\mathrm{FM}$ ground state.

In addition to coordination environment, the $\mathrm{Fe}^{3+}$ and $\mathrm{Fe}^{2+}$ ions are more accurately distinguished in $2 \mathrm{D} \mathrm{Fe}_{3} \mathrm{GeTe}_{2}$ by their different electronic behavior, which is confirmed by the charge density distributions in Fig. $\mathbf{1 b}$ and Bader charge on Fe atoms. ${ }^{23}$ Around the $\mathrm{Fe}^{2+}$ ions, electrons are more localized than that around the $\mathrm{Fe}^{3+}$ sites. The electrons are localized between $\mathrm{Fe}^{2+}$ and $\mathrm{Ge} / \mathrm{Te}$ ions, reflecting their covalent bonding characteristics. In comparison, more delocalized ionic bonding takes place between $\mathrm{Fe}^{3+}$ and $\mathrm{Ge} / \mathrm{Te}$ ions. This difference between localized and delocalized electron distributions around the $\mathrm{Fe}^{3+}$ and $\mathrm{Fe}^{2+}$ ions is also supported by Bader charge analysis. There is a net charge transfer of about 0.4 electrons from $\mathrm{Fe}^{3+}$ ions to 

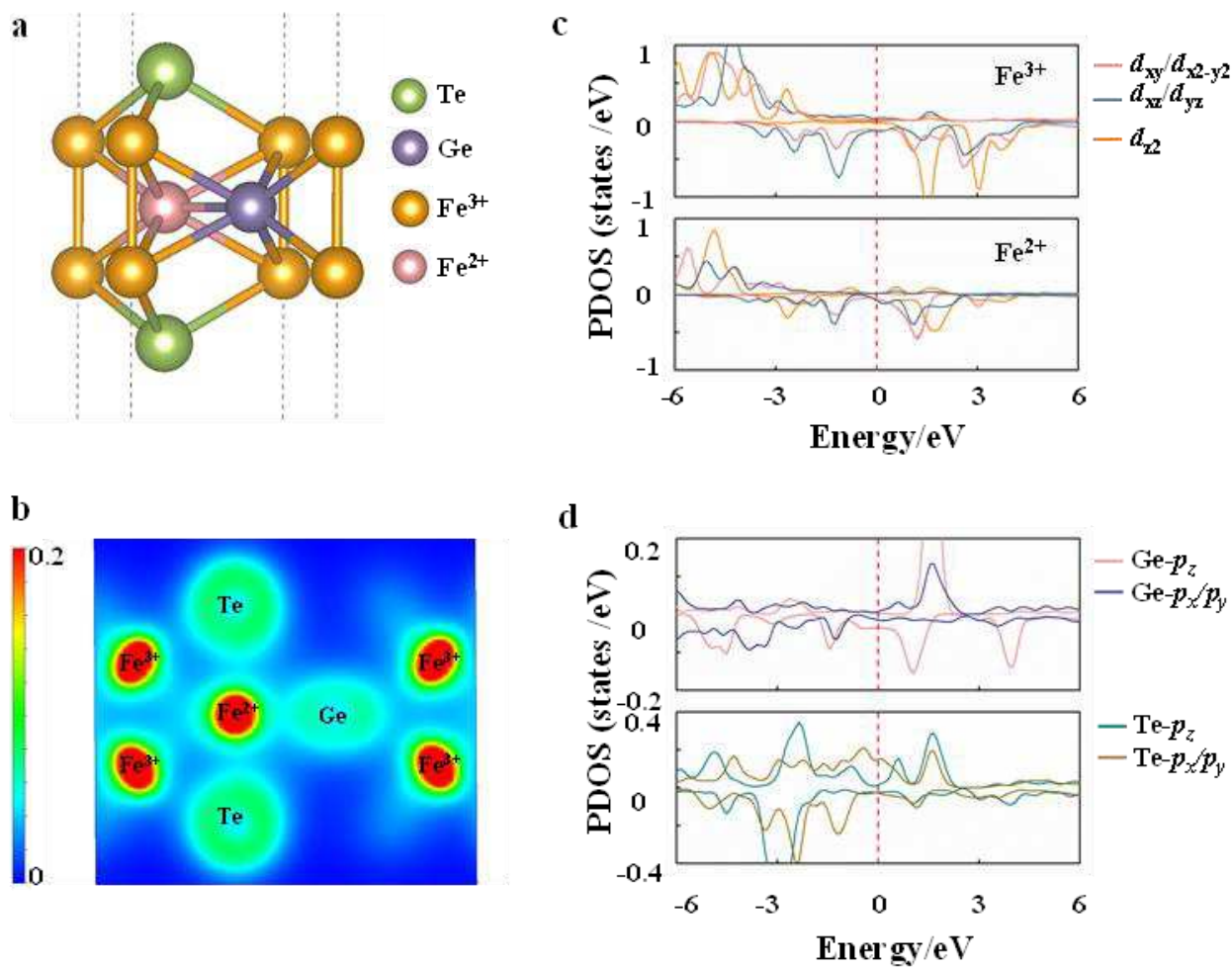

Fig. 1 Crystal and electronic structure of $2 \mathrm{D} \mathrm{Fe}_{3} \mathrm{GeTe}_{2}$. a A schematic plot of the structure. The gold, pink, purple and green balls stand for $\mathrm{Fe}^{3+}, \mathrm{Fe}^{2+}$, Te and $\mathrm{Ge}$ ions. b Charge density for $\mathrm{Fe}_{3} \mathrm{GeTe}_{2}$ monolayer on the (110) face. $\mathbf{c}$ The orbital-projected density of states (PDOS) for $\mathrm{Fe}, \mathrm{Ge}$ and Te atom, respectively. Among them, $\mathrm{Fe}$ atom could divide by trivalent iron $\mathrm{Fe}^{3+}$ and divalent iron $\mathrm{Fe}^{2+}$ The Fermi level (red dash line) is set to zero.

its surrounding $\mathrm{Ge} / \mathrm{Te}$ ions. However, there is no evident charge transfer in the case of $\mathrm{Fe}^{2+}$ ions. Following this picture, we reveal the $\mathrm{Fe}^{2+}$ ion is localized relative to $\mathrm{Fe}^{3+}$ ion in $2 \mathrm{D} \mathrm{Fe}_{3} \mathrm{GeTe}_{2}$, consistent with a previous report by Deiseroth et al. ${ }^{24}$ The Co-existence of localized and itinerant magnetism have also been found in the iron-based superconductors and double perovskite materials, such as $\mathrm{LaOFeAs}, \mathrm{Sr}_{2} \mathrm{FeMoO}_{6}$ and $\mathrm{La}_{1-\mathrm{x}} \mathrm{Sr}_{\mathrm{x}} \mathrm{MO}_{3}(\mathrm{M}=\mathrm{Mn}$ and $\mathrm{Co}){ }^{25,26,27 \text {, }}$ ${ }^{28}$, which all are polyvalence materials.

The partial density of states (PDOS) can further clarify the origin of different electronic and magnetic features in $\mathrm{Fe}^{3+}$ and $\mathrm{Fe}^{2+}$ ions of $2 \mathrm{D} \mathrm{Fe}_{3} \mathrm{GeTe}_{2}$, which shown in Fig. 1. Under a hexagonal crystal field, the five $3 d$ orbitals of Fe atom split into a single state $a_{1}\left(d_{z 2}\right)$, two twofold degenerate states $\mathrm{e}_{1}\left(d_{x z-y 2} / d_{x y}\right)$ and $\mathrm{e}_{2}\left(d_{x z} / d_{y z}\right)$. From the PDOS of Fe atoms, one can 
see that the $d_{z 2}$ orbital compared to the other $3 d$ orbitals is obviously narrow and sharp that suggested a localized feature. However, the $d_{x z-y 2}, d_{x y}, d_{x z}$, and $d_{y z}$ orbitals in the minority-spin channels are obviously wide and them hybridized with Ge/Te-p states, indicating the delocalized feature. Similar to $\mathrm{LaOFeAs}$, the localized $d$-electrons different from the itinerant electrons as coming from more isolated $d_{\mathrm{z} 2}$ orbitals. ${ }^{25}$ Moreover, $3 d$ bands of the majority spin for both $\mathrm{Fe}^{3+}$ and $\mathrm{Fe}^{2+}$ ions are fully occupied, while those of the minority spin are partially occupied. These results indicate that both $\mathrm{Fe}^{3+}$ and $\mathrm{Fe}^{2+}$ ions are in their high-spin configurations. The main difference between $\mathrm{Fe}^{3+}$ and $\mathrm{Fe}^{2+}$ ions on PDOS is due to the $d_{z 2}$ and $d_{x z} / d_{y z}$ states in the minority-spin channels. Specifically, the electron occupation number on minority $d_{z 2}$ orbital are 0.04 and 0.30 for $\mathrm{Fe}^{3+}$ and $\mathrm{Fe}^{2+}$ ions, respectively. That is to say, $\mathrm{Fe}^{2+}$ ion has $\sim 0.3$ more electron than $\mathrm{Fe}^{3+}$ ion to occupy the minority $d_{z 2}$ orbital. The occupied minority $d_{z 2}$ orbital results in $\mathrm{Fe}^{2+}$ ion is more localized than $\mathrm{Fe}^{3+}$ ion. In addition, compared to $\mathrm{Fe}^{2+}$ ion the energy level of minority $d_{x z} / d_{y z}$ states of $\mathrm{Fe}^{3+}$ ion will shift to the lower energy region. The corresponding number of occupied minority $d_{x z} / d_{y z}$ states increase from 0.35 to 0.54 . Therefore, the resulting calculating net magnetic moment is $3.0 \mu_{\mathrm{B}}$ and $2.6 \mu_{\mathrm{B}}$ for $\mathrm{Fe}^{3+}$ and $\mathrm{Fe}^{2+}$ ions, respectively. The localized $\mathrm{a}_{1}$ and delocalized $\mathrm{e}_{1} / \mathrm{e}_{2}$ states results in the unique magnetism in $2 \mathrm{D} \mathrm{Fe}_{3} \mathrm{GeTe}_{2}$, which possessed the coexistence of local and itinerant magnetism, consistent with a previous report by Yang et al. ${ }^{22}$ Obviously, the feature of localized magnetism play a crucial role in electronic and magnetism of metallic ferromagnetic $\mathrm{Fe}_{3} \mathrm{GeTe}_{2}$ system.

\section{Magnetic coupling mechanism and Curie temperatures of 2D $\mathrm{Fe}_{3} \mathrm{GeTe}_{2}$}

Because of the coexistence of localized and itinerant magnetism, the magnetic behavior of metallic ferromagnetic $\mathrm{Fe}_{3} \mathrm{GeTe}_{2}$ will deviate from itinerant Stoner model. Recently experiment indeed confirmed that metallic $\mathrm{Fe}_{3} \mathrm{GeTe}_{2}$ shown non-Stoner ferromagnetism. Yang et al. do not observe a considerable change in the electronic structure with temperature, which is not consistent with the expectation. Within the itinerant Stoner model, the ferromagnetic metal will exhibit a temperature-dependent exchange splitting that disappears above $\mathrm{T}_{\mathrm{C}}{ }^{22}$ Moreover, Tovar et al. used corrected Stoner parameter to describe the magnetic behaver in polyvalence $\mathrm{Sr}_{2} \mathrm{FeMoO}_{6}$, which also shown the coexistence of localized and itinerant magnetism. The corrections for Landau diamagnetism to Stoner parameter need to derived by experiment 
$\mathbf{a}$
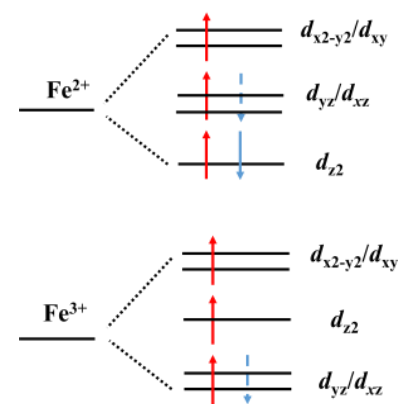

b

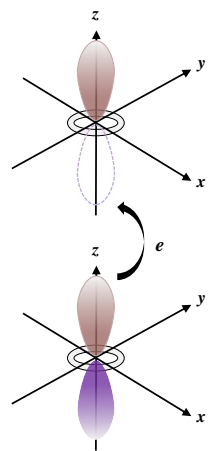

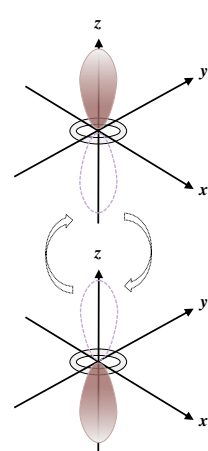

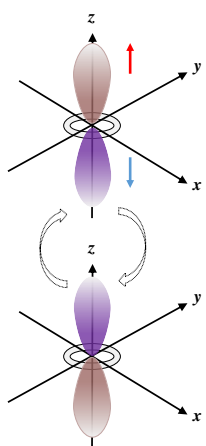

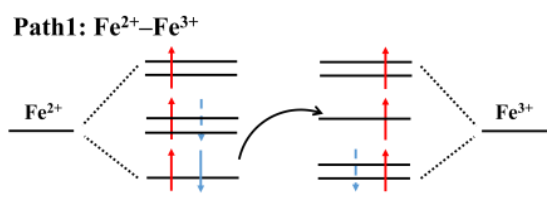

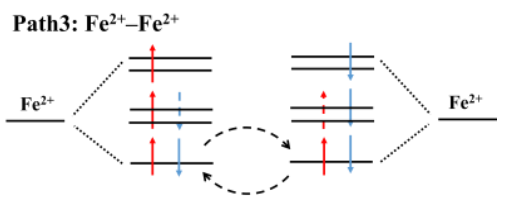

Path2: $\mathrm{Fe}^{3+}-\mathrm{Fe}^{3+}$

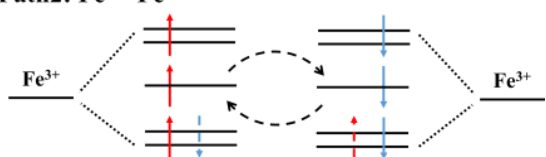

Fig. 2 The localized exchange in $2 \mathrm{D} \mathrm{Fe}_{3} \mathrm{GeTe}_{2}$. a The schematic representation of splitting $d$ orbital in $\mathrm{Fe}^{2+}$ and $\mathrm{Fe}^{3+}$ ions, respectively. $\mathbf{b}$ Three possible process of the exchange between localized $d_{\mathrm{z} 2}$ orbital c The corresponding three possible $\mathrm{Fe}-\mathrm{Fe}$ exchange paths in $\mathrm{Fe}_{3} \mathrm{GeTe}_{2}$ crystal.

measured. ${ }^{26}$ Therefore, we need a new model to describe the complicated ferromagnetism in $\mathrm{Fe}_{3} \mathrm{GeTe}_{2}$ systems.

In present paper, two main magnetic exchange mechanism have been introduced in $2 \mathrm{D}$ $\mathrm{Fe}_{3} \mathrm{GeTe}_{2}$. The itinerant magnetism between $\mathrm{e}_{1}$ and $\mathrm{e}_{2}$ electrons and localized magnetism in $\mathrm{a}_{1}$ spins. Therefore, we propose a multipath magnetic interaction mechanism to understand the localized magnetic exchange for $2 \mathrm{D} \mathrm{Fe}_{3} \mathrm{GeTe}_{2}$. According to the splitting of $\mathrm{Fe}^{2+}$ and $\mathrm{Fe}^{3+}$ orbitals in the crystal filed and multilayer structure of $2 \mathrm{D} \mathrm{Fe}_{3} \mathrm{GeTe}_{2}$, three possible exchange paths are considered. Fig. 2 shown the exchange paths between unoccupied $d_{z 2}$ orbital $\left(\mathrm{Fe}^{3+}-\right.$ $\left.\mathrm{Fe}^{3+}\right)$, occupied $d_{z 2}$ orbital $\left(\mathrm{Fe}^{2+}-\mathrm{Fe}^{2+}\right)$ and unoccupied to occupied $d_{z 2}$ orbital $\left(\mathrm{Fe}^{2+}-\mathrm{Fe}^{3+}\right)$, respectively. The hopping from the occupied Fe- $d_{z 2}$ orbitals to the unoccupied Fe- $d_{z 2}$ orbitals 
induces extremely strong FM coupling (path $\boldsymbol{P}_{1}$ ), which occurs between the magnetic ions in different oxidation states, namely double exchange. ${ }^{29}$ Double exchange play an essential role in polyvalence ferromagnetic materials like $\mathrm{La}_{1-\mathrm{x}} \mathrm{Sr}_{x} \mathrm{MnO}_{3}$ that also exhibited both localized and itinerant magnetism. ${ }^{30}$ However, spin crossover between both unpaired Fe- $d_{z 2}$ orbitals (path $\boldsymbol{P}_{2}$ ) and paired Fe- $d_{z 2}$ orbitals (path $\boldsymbol{P}_{\mathbf{3}}$ ) gives rise to an AFM interaction according to the Pauli exclusion principle. On the other hand, the non-spin-polarized PDOS (see Supplementary Fig. 3) shown that $d_{x z} / d_{y z}$ and $d_{x z-y 2} / d_{x y}$ mainly contributed at the fermi level, and the lower kinetic energy makes them contributed to the itinerant ferromagnetism in $2 \mathrm{D} \mathrm{Fe} \mathrm{GeTe}_{2}$. Therefore, the interaction between itinerant electrons in $\mathrm{e}_{1}$ states endow the intralayer FM $\left(\boldsymbol{I}_{1}\right)$, while the coupling between electrons in $\mathrm{e}_{2}$ states favor in interlayer FM $\left(\boldsymbol{I}_{2}\right)$. Among them, the intralayer FM only contributed by itinerant electrons $\left(\boldsymbol{I}_{\mathbf{1}}\right)$, but there is a competition between interlayer FM $\left(\boldsymbol{P}_{1}\right.$ and $\left.\boldsymbol{I}_{2}\right)$ and AFM $\left(\boldsymbol{P}_{2}\right.$ and $\left.\boldsymbol{P}_{3}\right)$ coupling. This explain why a debate regarding Fe atoms ferromagnetically or antiferromagnetically in $\mathrm{Fe}_{3} \mathrm{GeTe}_{2} \cdot{ }^{31}$ Moreover, $\mathrm{Fu}$ et al. also founded that the coexistence of localized and itinerant $3 d$ electrons in $\mathrm{BiFeO}_{3} / \mathrm{SrTiO}_{3}$ superlattices and itinerant Fe-3 $d$ electrons tends to cause ferromagnetism. ${ }^{32}$

A local Heisenberg model can provides a good description of the ferromagnetic ordering in $\mathrm{Fe}_{3} \mathrm{GeTe}_{2}$ system. ${ }^{14}$ In $2 \mathrm{D} \mathrm{Fe} \mathrm{GeTe}_{2}$, there are three types of exchange interaction between Fe ions that correspond to the first, second and third nearest neighboring magnetic exchange constants $J_{1}, J_{2}$ and $J_{3}$, as shown in Fig. 3a. The value of $J_{1}, J_{2}$ and $J_{3}$ for $2 \mathrm{D} \mathrm{Fe}_{3} \mathrm{GeTe}_{2}$ are extracted from the total energy difference between different spin orderings. As summarized in Table 1, the derived exchange interaction parameters are $J_{1}=-0.44 \mathrm{meV}, J_{2}=3.27 \mathrm{meV}$, and $J_{3}=0.47 \mathrm{meV}$. It is known that positive $J$ value favors FM ordering, while negative $J$ value favors AFM coupling. Therefore, the calculated $J_{1}$ of $-0.44 \mathrm{meV}$ yields weak AFM coupling, which occurs mainly through $\boldsymbol{P}_{\mathbf{2}}$ path. The path $\boldsymbol{P}_{\mathbf{1}}$ endows $J_{2}$ strong FM coupling with the value of $3.27 \mathrm{meV}$. Moreover, itinerant magnetism $\boldsymbol{I}_{\mathbf{1}}$ and $\boldsymbol{I}_{\mathbf{2}}$ making $J_{3}(0.47 \mathrm{meV})$ a long-range FM coupling. The coincidence between the magnetic interaction parameters and the effect of coexisting localized and itinerant magnetism, suggesting our proposed magnetic interaction mechanism is viable for understanding the magnetic ground state of $2 \mathrm{D} \mathrm{Fe}_{3} \mathrm{GeTe}_{2}$. Its reliability is also verified by the other theoretical report. For example, first-principles calculations by $\mathrm{Hu}$ et al. have shown that the stability of ferromagnetism can be largely enhanced by tensile strain 

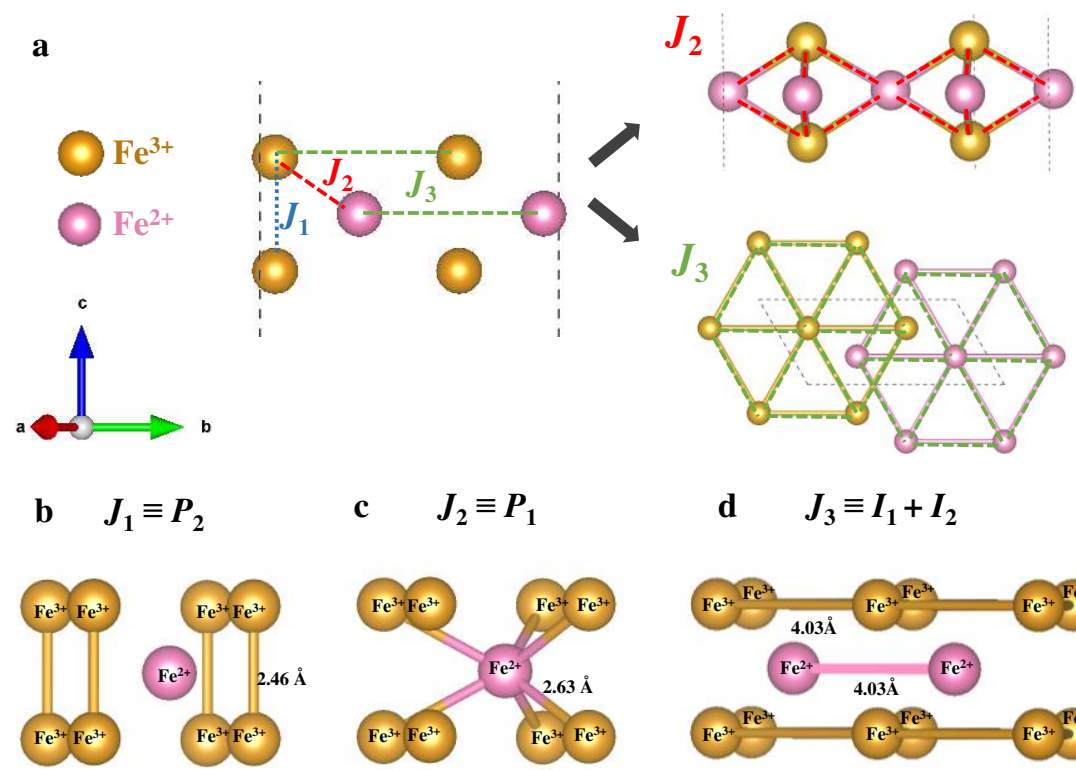

c $\quad J_{2} \equiv P_{1}$

d $\quad J_{3} \equiv I_{1}+I_{2}$
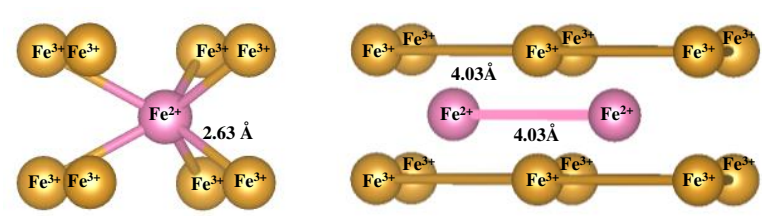

Fig. 3 Magnetic exchange parameter for $2 \mathrm{D} \mathrm{Fe}_{3} \mathbf{G e T e}_{2}$. a The side view shows $J_{1}$ to $J_{3}$ for the $\mathrm{Fe}-\mathrm{Fe}$ coupling in $\mathrm{Fe}_{3} \mathrm{GeTe}_{2}$ crystal. $\mathrm{Fe}-\mathrm{Fe}$ exchange interaction paths for magnetic exchange constants in monolayer $\mathrm{Fe}_{3} \mathrm{GeTe}_{2}$. b-d The schematic diagrams of exchange parameters $J_{1}, J_{2}$ and $J_{3}$, respectively.

in $\mathrm{Fe}_{3} \mathrm{GeTe}_{2}$ monolayer. ${ }^{33}$ Within our picture, tensile strain would shorten the $\mathrm{Fe}^{3+}-\mathrm{Fe}^{2+}$ distance ( $\boldsymbol{P}_{1}$ path) but elongate the other interatomic distances, which in turn enhance the FM double exchange between $\mathrm{Fe}^{3+}$ and $\mathrm{Fe}^{2+}$ ions.

MAE as an important parameter of ferromagnets counteracts thermal fluctuations and preserves the long-range ferromagnetic ordering. ${ }^{34}$ From non-collinear calculations with inclusion of the SOC effect, the MAE of $2 \mathrm{D} \mathrm{Fe}_{3} \mathrm{GeTe}_{2}$ is determined as $0.94 \mathrm{meV} / \mathrm{Fe}$, favoring perpendicular anisotropy, while the previously reported value was $0.67 \mathrm{meV} / \mathrm{Fe} .{ }^{14}$ For comparison, the MAE of $2.5 \mathrm{meV} / \mathrm{Fe}$ in bulk $\mathrm{Fe}_{3} \mathrm{GeTe}_{2}$ is slightly higher. The physical origin of positive MAE can be ascribed to the matrix element differences between the occupied and unoccupied spin-down $d$ orbitals of Fe atom. ${ }^{35}$ Roughly speaking, the coupling between occupied and unoccupied spin-down $d$ orbitals of Fe atoms contribute to the major part of MAE. By decomposing the contribution from each pair of states, the MAE of $2 \mathrm{D} \mathrm{Fe}_{3} \mathrm{GeTe}_{2}$ depend on the interaction between the $\mathrm{a}_{1}$ and $\mathrm{e}_{2}$ states. The positive contributions to MAE originate mainly 
Table 1. The $\mathrm{Fe}^{2+} / \mathrm{Fe}^{3+}$ ratio $(x)$, effective thickness, formation energy ( $\mathrm{E}_{\text {form }}$ ), magnetic moment $(M)$ per Fe atom, magnetic anisotropy energy (MAE) per unit cell, Curie temperature $\left(\mathrm{T}_{\mathrm{C}}\right)$, and exchange parameters $\left(J_{1}, J_{2}, J_{3}\right)$ of $\mathrm{Fe}_{n} \mathrm{GeTe}_{2}$ multilayer films.

\begin{tabular}{|c|c|c|c|c|c|c|c|c|c|}
\hline & \multirow[t]{2}{*}{$x$} & \multirow[t]{2}{*}{$\begin{array}{l}\text { Thickness } \\
\text { (̊̊) }\end{array}$} & \multirow[t]{2}{*}{$\begin{array}{c}\mathbf{E}_{\text {form }} \\
(\mathrm{eV} / \mathrm{atom})\end{array}$} & \multirow[t]{2}{*}{$\begin{array}{c}M \\
\left(\mu_{\mathrm{B}}\right)\end{array}$} & \multirow[t]{2}{*}{$\begin{array}{l}\text { MAE } \\
(\mathrm{meV})\end{array}$} & \multirow[t]{2}{*}{$\begin{array}{l}\mathbf{T}_{\mathbf{C}} \\
(\mathbf{K})\end{array}$} & \multicolumn{3}{|c|}{$\begin{array}{c}\text { Exchange parameter } \\
\qquad(\mathrm{meV})\end{array}$} \\
\hline & & & & & & & $J_{1}$ & $J_{2}$ & $J_{3}$ \\
\hline $\mathrm{Fe}_{3} \mathrm{GeTe}_{2}$ & 0.5 & 8.61 & -0.08 & 2.87 & 2.83 & 138 & -0.44 & 3.27 & 0.47 \\
\hline $\mathrm{Fe}_{4} \mathrm{GeTe}_{2}$ & 1.0 & 9.10 & -0.03 & 2.73 & -5.01 & 68 & 7.40 & 10.50 & 0.10 \\
\hline $\mathrm{Fe}_{5} \mathrm{GeTe}_{2}$ & 0 & 10.95 & -0.11 & 3.18 & 4.53 & 320 & -0.20 & 4.70 & -2.60 \\
\hline $\mathrm{Fe}_{6} \mathrm{GeTe}_{2}$ & 0.2 & 11.03 & -0.05 & 2.94 & 6.31 & 450 & 6.60 & -8.60 & 21.20 \\
\hline $\mathrm{Fe}_{7} \mathrm{GeTe}_{2}$ & 0.75 & 12.21 & -0.01 & 2.91 & 3.91 & 570 & -29.40 & 23.00 & 27.50 \\
\hline
\end{tabular}

from unoccupied $d_{z 2}$ orbital and half-occupied $d_{x z} / d_{y z}$ orbitals of $\mathrm{Fe}^{3+}$ ions, while the coupling of occupied $d_{z 2}$ and unoccupied $d_{x z} / d_{y z}$ orbitals of $\mathrm{Fe}^{2+}$ ions exhibit the negative contribution to the MAE. Such mechanism also accounts for the variation of MAE for $\mathrm{Fe}_{3} \mathrm{GeTe}_{2}$ monolayer by decreasing $\mathrm{Fe}^{3+}$ content due to hole doping, as observed by Park et al. ${ }^{36}$ Based on the obtained magnetic exchange constants and MAE, the Curie temperature of $2 \mathrm{D} \mathrm{Fe}_{3} \mathrm{GeTe}_{2}$ is estimated using 2D Heisenberg model (see Supplementary Fig. 4a). The obtained $\mathrm{T}_{\mathrm{C}}$ value of $138 \mathrm{~K}$ coincides well with previous experimental values of about $68-130 \mathrm{~K}$ for $2 \mathrm{D} \mathrm{Fe}_{3} \mathrm{GeTe}_{2}{ }^{13,14}$ The temperature-dependent magnetic moments (i.e., M-T curves) for each type of $\mathrm{Fe}$ ions ( $\mathrm{Fe}^{3+}$ and $\mathrm{Fe}^{2+}$ ) in $\mathrm{Fe}_{3} \mathrm{GeTe}_{2}$ compounds are also presented in Supplementary Fig. 4b. One can see that magnetizations of both $\mathrm{Fe}^{3+}$ and $\mathrm{Fe}^{2+}$ sublattices indeed behave like ferromagnets.

\section{$\mathrm{Fe}_{\mathrm{n}} \mathrm{GeTe}_{2}$ ultrathin films with tunable thickness}

Additionally, most experimental phenomena about multilayer and bulk $\mathrm{Fe}_{3} \mathrm{GeTe}_{2}$ in previous reports could be explained by the coexistence states of localized and itinerant magnetism, signifying the validity of these magnetic interaction mechanisms. For example, the estimated $\mathrm{T}_{\mathrm{C}}$ for bulk $\mathrm{Fe}_{3} \mathrm{GeTe}_{2}$ crystal is $280 \mathrm{~K}$ (see Supplementary Fig. 5), which is comparable to the experimental value of $230 \mathrm{~K} \cdot{ }^{24}$ Experimental study by Hwang et al. found 
AFM coupling between pristine $\mathrm{Fe}_{3} \mathrm{GeTe}_{2}$ layer and oxidized $\mathrm{Fe}_{3} \mathrm{GeTe}_{2}$ layers. Their DFT calculations further revealed that such AFM coupling mainly originates from the oxygen atoms located at the interface of bilayer, while bilayer $\mathrm{Fe}_{3} \mathrm{GeTe}_{2}$ with oxygen atom adsorbed in the top or bottom sites still prefers FM state. ${ }^{37}$ Within our localized Fe-Fe exchange model, the mediated oxygen atoms could provide an oxygen-mediated $\boldsymbol{P}_{\mathbf{2}}$ path between two $\mathrm{Fe}_{3} \mathrm{GeTe}_{2}$ layers, which induces AFM coupling. Dai et al. reported a pressure-dependent phase diagram of $\mathrm{Fe}_{3} \mathrm{GeTe}_{2}$ thin flakes, and the magnetic transformation temperature from ferromagnetic to paramagnetic states is $203 \mathrm{~K}$ at $3.7 \mathrm{GPa}$ and $163 \mathrm{~K}$ at $7.3 \mathrm{GPa}$, respectively. Moreover, the $\mathrm{T}_{\mathrm{C}}$ shows an obviously descending trend from 4.0 GPa to 7.3 GPa because the reduction of local magnetic moment and increased of electronic itinerancy. ${ }^{21}$ In the one hand, the increased electronic itinerancy could weaken the localized double exchange ( $\boldsymbol{P}_{\mathbf{1}}$ path). In the other hand, by analysis of their structural characteristics, we noticed that the $\mathrm{Fe}^{3+}-\mathrm{Te}$ distance distinctly decreases before pressures of $7 \mathrm{GPa}$. The corresponding $\mathrm{Fe}^{3+}-\mathrm{Fe}^{3+}$ exchange though the Temediated $\boldsymbol{P}_{2}$ path is stronger. The weaken FM coupling and enhanced AFM coupling, resulting in $\mathrm{T}_{\mathrm{C}}$ is drastically reduced. Especially, the gate-tunable electrons sequentially fill the sub-bands origin from the $\mathrm{Fe} d_{\mathrm{z} 2}, d_{\mathrm{xz}}$ and $d_{\mathrm{yz}}$ orbitals, induced room-temperature ferromagnetism in $\mathrm{Fe}_{3} \mathrm{GeTe}_{2} \cdot{ }^{14} \mathrm{The}$ value of $\mathrm{T}_{\mathrm{C}}$ mainly depended on the interaction between $d_{\mathrm{z} 2}, d_{\mathrm{xz}}$ and $d_{\mathrm{yz}}$ orbitals, consisting with our previously discuss. Moreover, the behavior from itinerant to localized magnetism enhanced the $T_{C}$, indicating the $\mathrm{FM}$ coupling in $\mathrm{Fe}_{3} \mathrm{GeTe}_{2}$ are mainly comes from the localized double exchange ( $\boldsymbol{P}_{1}$ path).

The above discussions on the one hand demonstrate again the coexistence of itinerant and localized magnetism in $\mathrm{Fe}_{3} \mathrm{GeTe}_{2}$ system. On the other hand, the interlayer competition between localized exchange coupling (path $\boldsymbol{P}_{\mathbf{1}}, \boldsymbol{P}_{\mathbf{2}}$ and $\boldsymbol{P}_{\mathbf{3}}$ ) and itinerant electrons $\left(\boldsymbol{I}_{\mathbf{1}}\right.$ and $\left.\boldsymbol{I}_{\mathbf{2}}\right)$ is also crucial for the magnetic ground states, Curie temperature and MAE of $2 \mathrm{D} \mathrm{Fe} \mathrm{GeTe}_{2}$. Moreover, the $\mathrm{T}_{\mathrm{C}}$ was enhanced from $143 \mathrm{~K}$ to $226 \mathrm{~K}$ with the increase in Fe content from 2.75 to 3.10 in bulk $\mathrm{Fe}_{3-\mathrm{x}} \mathrm{GeTe}_{2}{ }^{38}$ indicating the $\mathrm{T}_{\mathrm{C}}$ is very sensitive to $\mathrm{Fe}$ content. These findings motivate us to explore new high-temperature $\mathrm{Fe}-\mathrm{Ge}-\mathrm{Te}$ systems with optimal $\mathrm{Fe}^{2+} / \mathrm{Fe}^{3+}$ ratio and thickness, which the valence of Fe ions are related the direction of MAE and the competition of localized and itinerant magnetism in $\mathrm{Fe}_{n} \mathrm{GeTe}_{2}$ system. To satisfy these requirements, we have designed a series of Fe-rich $\mathrm{Fe}_{n} \mathrm{GeTe}_{2}(4 \leq n \leq 7)$ ultrathin films with various thickness (Fig. 4), which could 


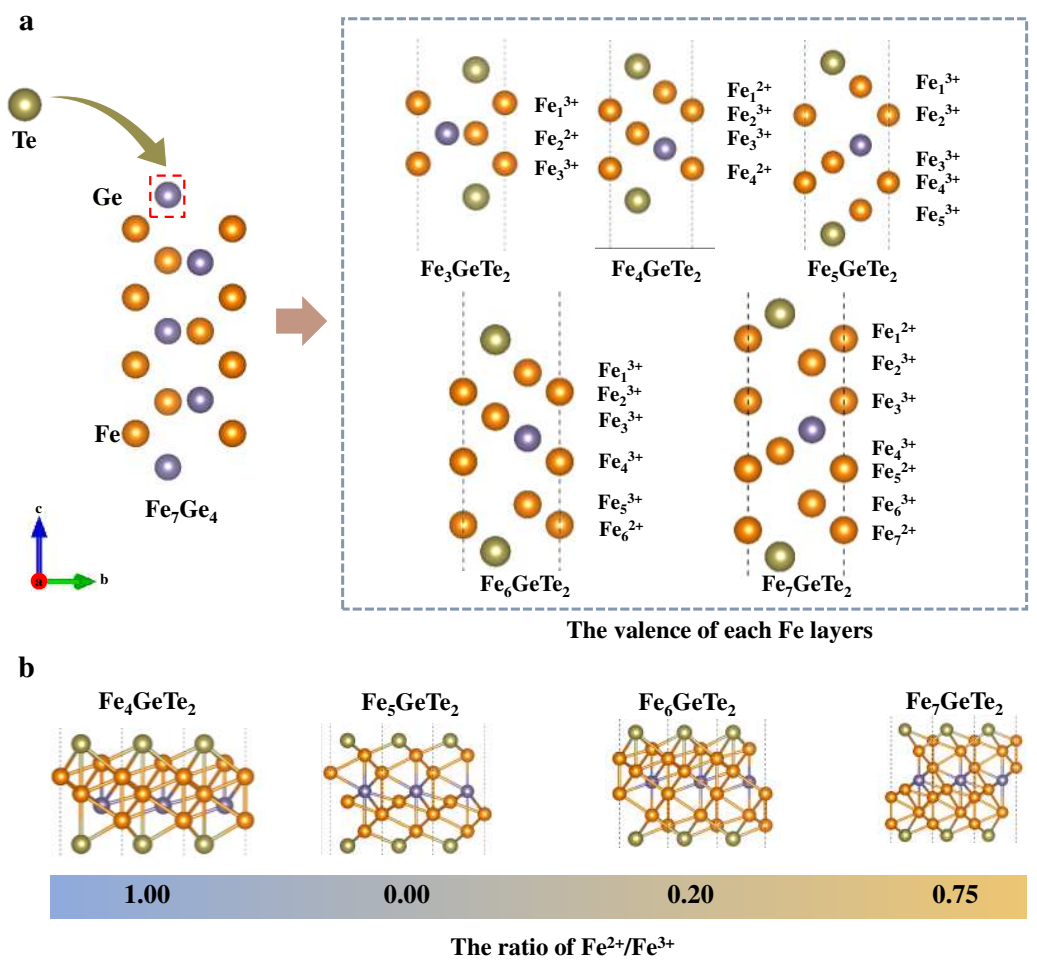

Fig. 4 Crystal strcture of $\mathrm{Fe}_{n} \mathrm{GeTe}_{2}(\mathbf{4} \leq \boldsymbol{n} \leq 7)$. a Schematic illustration of Te-substituted $\mathrm{Fe}_{7} \mathrm{Ge}_{4}$ crystal and constituted five structures in the series of $\mathrm{Fe}_{n} \mathrm{GeTe}_{2}$. b The stacked plane views of $\mathrm{Fe}_{\mathrm{n}} \mathrm{GeTe}_{2}$ multilayer films with difference $\mathrm{Fe}^{2+} / \mathrm{Fe}^{3+}$ ratio along [001] direction.

exhibit abundant magnetism by more complicated competition between itinerant and localized magnetism in multilayer structure. Similar to the $2 \mathrm{D} \mathrm{Fe}{ }_{3} \mathrm{GeTe}_{2}, \mathrm{Fe}_{\mathrm{n}} \mathrm{GeTe}_{2}$ ultrathin films also belong to $P-3 m 1$ space group. The effective thicknesses (Table 1) of $\mathrm{Fe}_{4} \mathrm{GeTe}_{2}, \mathrm{Fe}_{5} \mathrm{GeTe}_{2}$, $\mathrm{Fe}_{6} \mathrm{GeTe}_{2}, \mathrm{Fe}_{7} \mathrm{GeTe}_{2}$ ultrathin films are $5.63 \AA, 6.79 \AA, 7.56 \AA$, and $8.73 \AA$, respectively, which are moderately larger than that of $\mathrm{Fe}_{3} \mathrm{GeTe}_{2}(5.14 \AA)$. The atomic arrangement of $\mathrm{Fe}_{n} \mathrm{GeTe}_{2}$ ultrathin films can be regarded as six, seven, eight, and nine atomic layered thickness (001) surface of Te-substituted $\mathrm{Fe}_{7} \mathrm{Ge}_{4}$ crystal. ${ }^{39}$ Fortunately, the atomic arrangement of five atomic layered thickness Te-substituted $\mathrm{Fe}_{7} \mathrm{Ge}_{4}$ crystal is the same as the experimentally reported $\mathrm{Fe}_{3} \mathrm{GeTe}_{2}$ phase. To further check the experimentally feasibility of $\mathrm{Fe}_{n} \mathrm{GeTe}_{2}$, we calculated their formation energies, which is defined as

$$
E_{\mathrm{f}}=\left[E\left(\mathrm{Fe}_{\mathrm{n}} \mathrm{GeTe}_{2}\right)-E\left(\mathrm{Fe}_{2} \mathrm{Ge}\right)-E\left(\mathrm{Te}_{2}\right)-(\mathrm{n}-3) E(\mathrm{Fe})\right] /(\mathrm{n}+3)
$$


where $E\left(\mathrm{Fe}_{\mathrm{n}} \mathrm{GeTe}_{2}\right)$ represents the total energy of $2 \mathrm{D} \mathrm{Fe} \mathrm{HeTe}_{2}$ compound, and $E\left(\mathrm{Fe}_{2} \mathrm{Ge}\right), E\left(\mathrm{Te}_{2}\right)$ and $E(\mathrm{Fe})$ are the total energy for $\mathrm{Fe}_{2} \mathrm{Ge}$, $\mathrm{Te}$ and $\mathrm{Fe}$ in their most stable bulk phase, respectively. ${ }^{40}$ The formation energies of four $\mathrm{Fe}_{\mathrm{n}} \mathrm{GeTe}_{2}$ ultrathin films from our theoretical design are $-0.03 \mathrm{eV} /$ atom $(\mathrm{n}=4),-0.11 \mathrm{eV} /$ atom $(\mathrm{n}=5),-0.05 \mathrm{eV} /$ atom $(\mathrm{n}=6)$, and -0.01 $\mathrm{eV} / \mathrm{atom}(\mathrm{n}=7)$, respectively, which are comparable to the formation energy of $-0.08 \mathrm{eV} /$ atom for $\mathrm{Fe}_{3} \mathrm{GeTe}_{2}$. All these negative values indicate that their formation processes are exothermic. More importantly, we find that the total energy of our proposed $\mathrm{Fe}_{5} \mathrm{GeTe}_{2}$ ultrathin film is 0.24 $\mathrm{eV}$ per atom lower than that of the experimentally reported layered phase with same stoichiometry. ${ }^{16}$ The satisfactory stability of these $\mathrm{Fe}_{n} \mathrm{GeTe}_{2}$ ultrathin film implies that they are feasible from the theoretical point of view. It is noteworthy that ultrathin films of $\mathrm{Cr}_{2} \mathrm{~S}_{3}, \mathrm{CrSe}$ and FeTe in FM state have been synthesized by CVD and MBE methods in previous experiments. ${ }^{11,12,41}$

\section{Magnetic behavior of $\mathrm{Fe}_{\mathrm{n}} \mathrm{GeTe}_{2}$ ultrathin films}

We further discuss the electronic and magnetic properties of the proposed $\mathrm{Fe}_{\mathrm{n}} \mathrm{GeTe}_{2}$ ultrathin films. Similar to $2 \mathrm{D} \mathrm{Fe}_{3} \mathrm{GeTe}_{2}$, all $\mathrm{Fe}_{\mathrm{n}} \mathrm{GeTe}_{2}$ systems are metallic, as seen from the electronic band structures in Supplementary Fig. 6. The orbital project densities of states in Supplementary Fig. 7 demonstrate that the metallicity still originate from $d$ orbitals of $\mathrm{Fe}$ atoms. The coexistence of itinerant and localized $d$ electrons in $\mathrm{Fe}_{n} \mathrm{GeTe}_{2}(3 \leq n \leq 7)$ could revealed by the Bader charge (see Supplementary Table 1) and PDOS. The distribution of $\mathrm{Fe}^{2+}$ and $\mathrm{Fe}^{3+}$ ions vary with the thickness and compositions of $2 \mathrm{D} \mathrm{Fe}_{\mathrm{n}} \mathrm{GeTe}_{2}$ ultrathin films. With increasing Fe content, the $\mathrm{Fe}^{2+} / \mathrm{Fe}^{3+}(x)$ ratio is $0.5,1.0,0,0.2$, and 0.75 for $\mathrm{n}=3,4,5,6$, and 7 , respectively, which represented a magnetic behave from itinerant to localized. In order to investigate the ground states of $\mathrm{Fe}_{\mathrm{n}} \mathrm{GeTe}_{2}$ ultrathin films, we considered $\mathrm{FM}$ and various AFM configurations (see Supplementary Fig. 8). Due to the multilayer structures, the considered AFM configurations are increased with the Fe content. From our DFT calculations, FM ordering in all $\mathrm{Fe}_{\mathrm{n}} \mathrm{GeTe}_{2}$ systems is more favorable than the AFM or NM counterparts. The magnetic moments as a function of $x$ is plotted in Fig. 5a. With increasing $\mathrm{Fe}^{2+} / \mathrm{Fe}^{3+}$ ratio, the average magnetic moments per Fe atom slightly declines from $3.18 \mu_{\mathrm{B}}$ for $x=0$ to $2.87 \mu_{\mathrm{B}}$ for $x=1$. This observation can be easily understood that $\mathrm{Fe}^{3+}$ ions contribute larger magnetic moment 

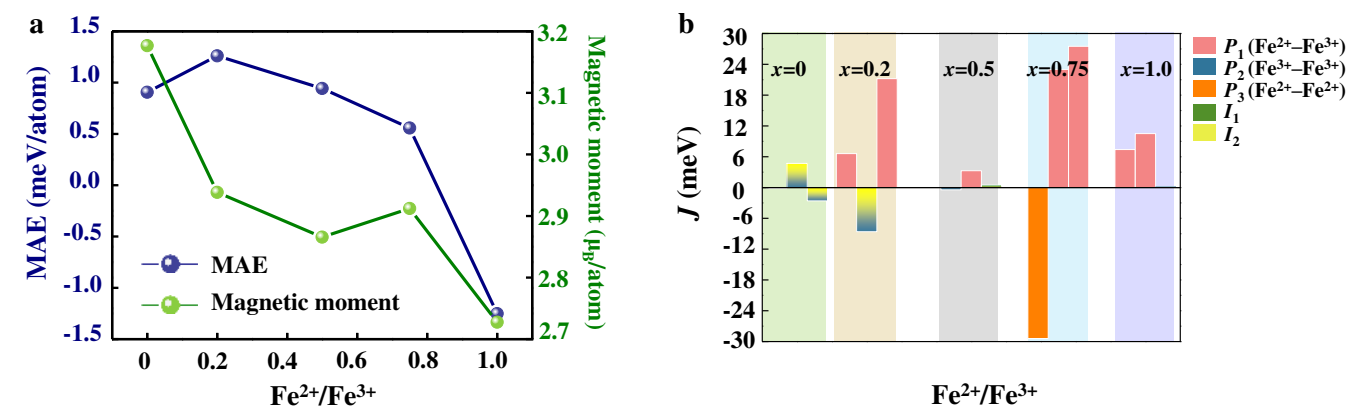

Fig. 5 Magnetic properties of multilayer $\mathrm{Fe}_{\mathrm{n}} \mathrm{GeTe}_{2}$. a The Calculated MAE and magnetic moment per atom at various $\mathrm{Fe}^{2+} / \mathrm{Fe}^{3+}$ ratio $(x)$. The detailed data are listed in Table 1. b The exchange parameters for first, second and third nearest neighbor (see Supplementary Fig. 9). The green, gold, gray, blue and purple regions represent $x=0,0.2,0.5,0.75$ and 1.0 , respectively.

than $\mathrm{Fe}^{2+}$ ions. In other words, the itinerant behavior of $d$ orbitals is weakened as the $\mathrm{Fe}^{2+} / \mathrm{Fe}^{3+}$ ratio increases.

Because the $\mathrm{T}_{\mathrm{C}}$ in $\mathrm{Fe}_{\mathrm{n}} \mathrm{GeTe}_{2}$ systems are mainly come from localized double exchange, we considered the exchange parameters $J$ of $\mathrm{Fe}_{n} \mathrm{GeTe}_{2}$ ultrathin film that are presented in Table 1 and Supplementary Fig. 9. Meanwhile, the long-range magnetic coupling with $J=5$ instead of $J=3$ exchange parameters has been considered in $\mathrm{Fe}_{\mathrm{n}} \mathrm{GeTe}_{2}$ ( $\mathrm{n}=5$ to 7 ) with the $\mathrm{Fe}$ content increased. (see Supplementary Table 2). Before the $\mathrm{Fe}^{2+} / \mathrm{Fe}^{3+}$ ratio up to 0.5 , their magnitude and sign is insensitive to the distance of magnetic ion pairs and obviously existed a competition between localized and itinerant magnetism. With the $\mathrm{Fe}^{2+} / \mathrm{Fe}^{3+}$ ratio continually increased, the localized magnetic exchange are dominant and their magnitude decreased with the distance of Fe ions. Meanwhile, the variations of $J_{1}, J_{2}$, and $J_{3}$ can be also interpreted by magnetic interaction mechanism, which has been established in the $2 \mathrm{D} \mathrm{Fe}_{3} \mathrm{GeTe}_{2}$ earlier.

To further clarify the magnetic ground states of $2 \mathrm{D} \mathrm{Fe}_{\mathrm{n}} \mathrm{GeTe}_{2}$ ultrathin films, the relationship between exchange path dependent parameters $J_{1}, J_{2}$, and $J_{3}$ with $\mathrm{Fe}^{2+} / \mathrm{Fe}^{3+}$ ratio $x$ is displayed in Fig. 5b, from which we can raise several arguments. First, due to the exchange through $d_{\mathrm{z} 2}$ and $d_{\mathrm{xz}} / d_{\mathrm{yz}}$ orbital are dominant in multilayer structures, the strength of interlayer localized $d_{\mathrm{z} 2}$ orbital interactions $\left(\boldsymbol{P}_{\mathbf{1}}, \boldsymbol{P}_{\mathbf{2}}\right.$ and $\boldsymbol{P}_{\mathbf{3}}$ paths $)$ and itinerant electrons coupling of $d_{\mathrm{xz}} / d_{\mathrm{yz}}$ orbital $\left(\boldsymbol{I}_{2}\right)$ prevail over the intralayer interactions $\left(\boldsymbol{I}_{\mathbf{1}}\right.$ path) in $\mathrm{Fe}_{\mathrm{n}} \mathrm{GeTe}_{2}$ systems. Secondly, for 
all the $\mathrm{Fe}_{\mathrm{n}} \mathrm{GeTe}_{2}$ systems considered here, the dominant $J$ parameter for FM coupling mainly comes from double exchange of localized $d_{\mathrm{z} 2}$ orbital $\left(\boldsymbol{P}_{\mathbf{1}}\right.$ path $)$ and itinerant electrons of $d_{\mathrm{xz}} / d_{\mathrm{yz}}$ orbitals coupling $\left(\boldsymbol{I}_{\mathbf{2}}\right)$. But the major $J$ parameter for AFM ordering is mainly from the couplings between the localized $d_{\mathrm{z} 2}$ orbitals in $\mathrm{Fe}^{3+}-\mathrm{Fe}^{3+}$ and $\mathrm{Fe}^{2+}-\mathrm{Fe}^{2+}$ exchange ( $\boldsymbol{P}_{\mathbf{2}}$ and $\boldsymbol{P}_{\mathbf{3}}$ paths). Therefore, the competition between interlayer AFM and FM coupling derived from the struggle between the itinerant and localized magnetism in $\mathrm{Fe}^{2+}-\mathrm{Fe}^{2+}$ or $\mathrm{Fe}^{3+}-\mathrm{Fe}^{3+}$ coupling. In $\mathrm{Fe}_{5} \mathrm{GeTe}_{2}$ ultrathin film, when the distance of interlayer Fe layers is shortly the localized magnetic exchange through $\boldsymbol{P}_{2}$ path could compete with itinerant $\mathrm{e}_{2}$ electrons. However, the itinerant $\mathrm{e}_{2}$ electrons become dominant with the $\mathrm{Fe}-\mathrm{Fe}$ distance increased such as the value of $J_{4}$ is $5.9 \mathrm{meV}$. Subsequently, the itinerant magnetism weakens with the $\mathrm{Fe}-\mathrm{Fe}$ distance continued increased, which the value of $J_{5}$ is $0.1 \mathrm{meV}$. Two competing ferromagnetism of localized and itinerant are responsible for these complicated behavior of the magnetic exchange parameters. $\mathrm{As} \mathrm{Fe}^{2+} / \mathrm{Fe}^{3+}$ ratio increases, more localized $\boldsymbol{P}_{1} / \boldsymbol{P}_{3}$ path $(\mathrm{FM} / \mathrm{AFM})$ and less $\boldsymbol{I}_{2}(\mathrm{FM})$ could be introduced; thus there is no simple trend for the variation of $J$ parameters.

The MAE values for all the $\mathrm{Fe}_{n} \mathrm{GeTe}_{2}$ ultrathin films with different $\mathrm{Fe}^{2+} / \mathrm{Fe}^{3+}$ ratios are also shown in Fig. 5a. One can see that MAE first increases from $0.91 \mathrm{meV} / \mathrm{Fe}$ atom for $x=0$ $\left(\mathrm{Fe}_{5} \mathrm{GeTe}_{2}\right)$ to $1.05 \mathrm{meV} / \mathrm{Fe}$ atom for $x=0.2\left(\mathrm{Fe}_{6} \mathrm{GeTe}_{2}\right)$. Then, it almost monotonically decreases with increasing $\mathrm{Fe}^{2+} / \mathrm{Fe}^{3+}$ ratio in the mixed-valence $\mathrm{Fe}_{n} \mathrm{GeTe}_{2}$ compounds. As the $x$ further increases to 1 , the easy axis would flip from perpendicular into in-plane orientation. The amplitude and direction of magnetic anisotropy are affected by two competing factors simultaneously. One is the $\mathrm{Fe}^{2+} / \mathrm{Fe}^{3+}$ ratio. As we have discussed in $2 \mathrm{D} \mathrm{Fe}_{3} \mathrm{GeTe}_{2}, \mathrm{Fe}^{3+}$ and $\mathrm{Fe}^{2+}$ ions contribute to positive and negative MAE, respectively. Another important factor is the interaction between $d_{\mathrm{z} 2}$ and $d_{\mathrm{xz}} / d_{\mathrm{yz}}$ orbitals, since the electronic band structures reveal that the spin-minority components of these orbitals are affected by spin-orbit coupling associated with the inserted $\mathrm{Fe}$ layers. To further unveil the origin of MAE enhancement from $\mathrm{Fe}_{5} \mathrm{GeTe}_{2}$ to $\mathrm{Fe}_{6} \mathrm{GeTe}_{2}$, we decomposed the MAE to the coupling of $d_{z 2}$ and $d_{x z} / d_{y z}$ pairs by Eq.(2) (see Supplementary Fig. 10). When $\mathrm{Fe}^{2+} / \mathrm{Fe}^{3+}$ ratio is $0\left(\mathrm{Fe}_{5} \mathrm{GeTe}_{2}\right)$, there only exist positive contributions of occupied $d_{x z} / d_{y z}$ and unoccupied $d_{z 2}$ pair with the difference in orbital energy levels of about $4.08 \mathrm{eV}$, leading to an out-of-plane MAE of $0.91 \mathrm{meV} / \mathrm{Fe}$ atom. With $\mathrm{Fe}^{2+} / \mathrm{Fe}^{3+}$ ratio increases from $0\left(\mathrm{Fe}_{5} \mathrm{GeTe}_{2}\right)$ to $0.2\left(\mathrm{Fe}_{6} \mathrm{GeTe}_{2}\right)$, the energy level differences between 

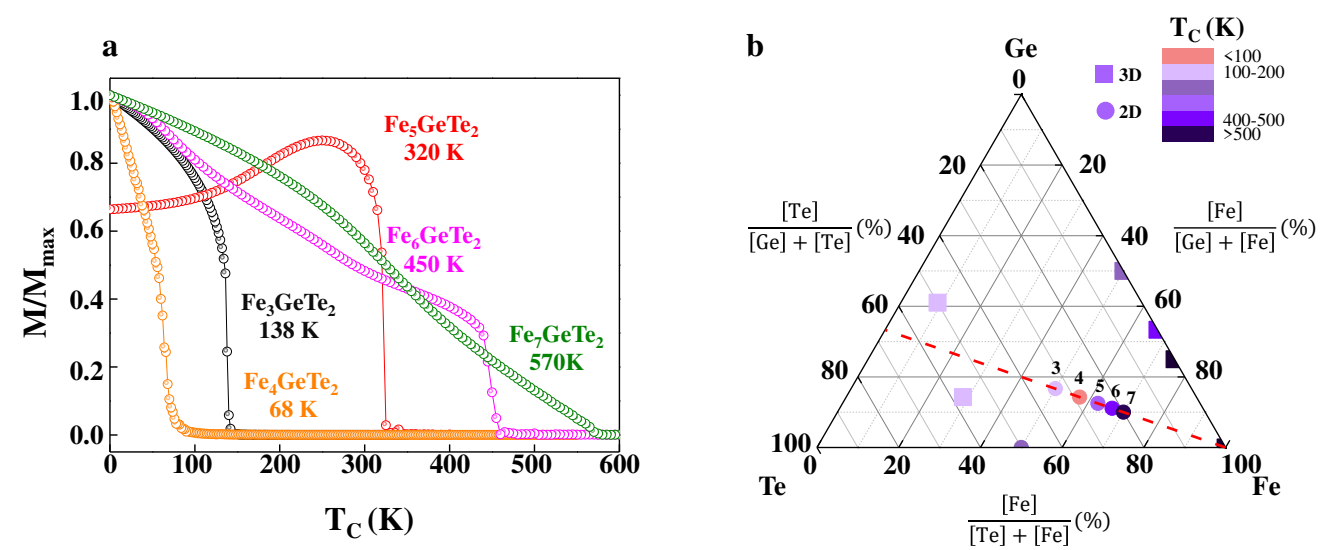

Fig. 6 Thickness-dependent magnetic properties of multilayer $\mathrm{Fe}_{n} \mathrm{GeTe}_{2}$ and comparison with other Fe-rich ferromagnets. a Calculated normalized magnetization of $\mathrm{Fe}$ atoms in $\mathrm{Fe}_{\mathrm{n}} \mathrm{GeTe}_{2}$ as a function of temperature from MC simulation. b Ternary phase diagram of various Fe-rich compositions, together with the stoichiometric line of Fe:Ge:Te $=\mathrm{n}: 1: 2(3 \leq n \leq 7)$. The color indicates the value of $\mathrm{T}_{\mathrm{C}}$. The squares and circles represent $3 \mathrm{D}$ and $2 \mathrm{D}$ strctures, respectively.

occupied $d_{x z} / d_{y z}$ and unoccupied $d_{z 2}$ pair and unoccupied $d_{x z} / d_{y z}$ and occupied $d_{z 2}$ pair are 3.79 and $4.65 \mathrm{eV}$, respectively. Therefore, for the occupied $d_{x z} / d_{y z}$ and unoccupied $d_{z 2}$ pair, the positive contributions to MAE prevail the negative contributions.

Using these exchange parameters and MAE strengths, we have evaluated the total M-T curves of $\mathrm{Fe}_{n} \mathrm{GeTe}_{2}$ systems, as shown in Fig. 6a. We have also simulated the M-T curves for every Fe sublattices (see Supplementary Fig. 11). For various stoichiometries, three kinds of M-T curves are observed. Similar to $\mathrm{Fe}_{3} \mathrm{GeTe}_{2}, \mathrm{Fe}_{4} \mathrm{GeTe}_{2}$ is also a true ferromagnet. The magnetic spin moments of all $\mathrm{Fe}$ atoms align in the same direction, and they decrease with increasing temperature, yielding a $\mathrm{T}_{\mathrm{C}}$ value of $68 \mathrm{~K}$. For $\mathrm{Fe}_{5} \mathrm{GeTe}_{2}$, the magnetic moment keeps increasing with temperature, and full compensation is not observed in the entire temperature range. The maximum in spontaneous magnetization appears between the $0 \mathrm{~K}$ and $\mathrm{T}_{\mathrm{C}}(320 \mathrm{~K})$. For each $\mathrm{Fe}$ sublattice, the $\mathrm{Fe}_{1}$ and $\mathrm{Fe}_{5}$ layers are thermally disturbed more easily and their magnetic moments almost decrease linearly with increasing temperature, while the other three layers $\left(\mathrm{Fe}_{2}, \mathrm{Fe}_{3}\right.$, and $\left.\mathrm{Fe}_{4}\right)$ drastically decrease around $320 \mathrm{~K}$. As a result, $\mathrm{Fe}_{5} \mathrm{GeTe}_{2}$ behaves as 
a Néel's P-type ferrimagnet. The ferrimagnetic to paramagnetic transition occurs at a critical temperature of $T_{C}=320 \mathrm{~K}$. According to Néel's classification of ferrimagnets, ${ }^{42} 2 \mathrm{D} \mathrm{Fe}_{6} \mathrm{GeTe}_{2}$ and $\mathrm{Fe}_{7} \mathrm{GeTe}_{2}$ sheets are the Néel's R-types. Their M-T curves exhibit monotonic reduction with increasing temperature. For the Fe sublattices of $\mathrm{Fe}_{6} \mathrm{GeTe}_{2}$, the spin moments of $\mathrm{Fe}_{1}, \mathrm{Fe}_{3}$, $\mathrm{Fe}_{4}, \mathrm{Fe}_{5}$, and $\mathrm{Fe}_{6}$ layers and $\mathrm{Fe}_{2}$ layers show opposite directions, while all the moments decrease with temperature. For the M-T curves of $2 \mathrm{D} \mathrm{Fe}_{7} \mathrm{GeTe}_{2}$, the spin directions of $\mathrm{Fe}_{2}, \mathrm{Fe}_{4}$ and $\mathrm{Fe}_{5}$ layers and $\mathrm{Fe}_{1}, \mathrm{Fe}_{3} \mathrm{Fe}_{6}$ and $\mathrm{Fe}_{7}$ layers are opposite. The ferrimagnetic to paramagnetic transition occurs at $\mathrm{T}_{\mathrm{C}}$ of $570 \mathrm{~K}$. The present Néel's R- and P-types of magnetization profiles have also been reported in mixed-valence complex alloys $\left(\mathrm{Mn}_{1.5} \mathrm{FeV}_{0.5} \mathrm{Al}{ }^{43}\right.$ and $\left.\mathrm{Mn}_{2} \mathrm{~V}_{0.5} \mathrm{C}_{0.5} \mathrm{Z}{ }^{44}\right)$, complex oxides $\left(\mathrm{NiCo}_{2} \mathrm{O}_{4}{ }^{45}\right)$, layered materials $\left(\mathrm{AFe}^{\mathrm{II}} \mathrm{Fe}^{\mathrm{III}}\left(\mathrm{C}_{2} \mathrm{O}_{4}\right)_{3}{ }^{46}\right)$ and core-shell nanoparticles ${ }^{47}$. The competition of interlayer AFM and FM coupling resulting in the transition from $\mathrm{FM}$ to FiM states in $\mathrm{Fe}_{\mathrm{n}} \mathrm{GeTe}_{2}$, deriving from the coexistence of different electronic states.

The simulated $\mathrm{T}_{\mathrm{C}}$ are $138 \mathrm{~K}$ for $\mathrm{Fe}_{3} \mathrm{GeTe}_{2}, 68 \mathrm{~K}$ for $\mathrm{Fe}_{4} \mathrm{GeTe}_{2}, 320 \mathrm{~K}$ for $\mathrm{Fe}_{5} \mathrm{GeTe}_{2}, 450 \mathrm{~K}$ for $\mathrm{Fe}_{6} \mathrm{GeTe}_{2}$, and $570 \mathrm{~K}$ for $\mathrm{Fe}_{7} \mathrm{GeTe}_{2}$, respectively. For the truly ferromagnetic systems, $\mathrm{T}_{\mathrm{C}}$ drops from $138 \mathrm{~K}$ for $\mathrm{Fe}_{3} \mathrm{GeTe}_{2}$ to $68 \mathrm{~K}$ for $\mathrm{Fe}_{4} \mathrm{GeTe}_{2}$ because of the flipping of out-of-plane MAE brought by the increased ratio of $\mathrm{Fe}^{2+}$ ions. Further increasing $\mathrm{Fe}$ content leads to a transition of magnetic ordering from ferromagnetic to ferrimagnetic at $\mathrm{Fe}_{5} \mathrm{GeTe}_{2}$. For $\mathrm{n} \geq 5, \mathrm{~T}_{\mathrm{C}}$ of the ferrimagnetic $\mathrm{Fe}_{\mathrm{n}} \mathrm{GeTe}_{2}$ film increases with $\mathrm{n}$, mainly due to higher MAE and stronger double exchange. The similar trend have also been observed in ferromagnetic $\mathrm{Fe}_{3-\mathrm{x}} \mathrm{Cr}_{\mathrm{x}} \mathrm{Ge}$ and $\mathrm{Fe}_{3-\mathrm{x}} \mathrm{V}_{\mathrm{x}} \mathrm{Ge}$ alloys. ${ }^{48,}{ }^{49}$ By extrapolating to even thicker films, $\mathrm{Fe}_{\mathrm{n}} \mathrm{GeTe}_{2}$ with $\mathrm{n}=9$ and an effective thickness of $14 \AA$ yields a $\mathrm{T}_{\mathrm{C}}=1006 \mathrm{~K}$, which is comparable to $\mathrm{T}_{\mathrm{C}}=1043 \mathrm{~K}$ for pure Fe solid of bcc phase ${ }^{15}$ (see Supplementary Fig. 12).

\section{Ternary phase diagram of $T_{C}$ for $F e$ based compounds}

In order to provide a more general aspect to the composition and dimensional effects on the magnetic behavior of $\mathrm{Fe}-\mathrm{Ge}-\mathrm{Te}$ systems, we plot a ternary phase diagram of $\mathrm{T}_{\mathrm{C}}$ for various reported Fe based compounds (Fig. 6b). In the three-dimensional (3D) compounds, the $\mathrm{T}_{\mathrm{C}}$ of Fe-rich composition monotonically increases from $279 \mathrm{~K}$ for $\mathrm{FeGe}^{50}$ to $485 \mathrm{~K}$ for $\mathrm{Fe}_{2} \mathrm{Ge}^{51}$, and then to $1043 \mathrm{~K}$ for pure $\mathrm{Fe},{ }^{15}$ showing a prominent composition effect. In the $2 \mathrm{D} \mathrm{Fe}-\mathrm{Ge}-\mathrm{Te}$ films, $\mathrm{T}_{\mathrm{C}}$ is determined by the combined composition and dimensional effects. Generally 
speaking, incorporation of $\mathrm{Fe}$ atoms in the system would increase $\mathrm{T}_{\mathrm{C}}$. For example, Fe doping generates the long-range spin ordering in GeTe films and the $\mathrm{T}_{\mathrm{C}}$ of $\mathrm{Fe}_{0.18} \mathrm{Ge}_{0.82} \mathrm{Te}$ films is 100 $\mathrm{K} .{ }^{52} \mathrm{The} \mathrm{T}_{\mathrm{C}}$ of our $\mathrm{Fe}_{3} \mathrm{GeTe}_{2}(138 \mathrm{~K})$ with effective thickness of $0.86 \mathrm{~nm}$ is lower than the FeTe ultrathin film with $2.80 \mathrm{~nm}\left(\mathrm{~T}_{\mathrm{C}}=220 \mathrm{~K}\right)^{12}$, even with the same Fe content. Moreover, the $\mathrm{T}_{\mathrm{C}}$ of $\mathrm{Fe}_{6} \mathrm{GeTe}_{2}(450 \mathrm{~K})$ is slightly lower to the bulk $\mathrm{Fe}_{2} \mathrm{Ge}$ phase $\left(\mathrm{T}_{\mathrm{C}}=485 \mathrm{~K}\right)$. Both of them have Fe content of 0.67. However, the role of non-metal element (Ge and $\mathrm{Te}$ ) inclusion in the ultrathin Fe-Ge-Te films is very complicated. They can not only tune the chemical valence state and the electronic behavior of the variable element Fe but also provide crystal field to control MAE, which will change the magnetic behave and $\mathrm{T}_{\mathrm{C}}$.

\section{Discussion}

We design a family of $2 \mathrm{D} \mathrm{Fe}_{\mathrm{n}} \mathrm{GeTe}_{2}$ ultrathin films with different Fe contents and thickness, which are experimentally more feasible than the reported 2D layered phase. By first-principle calculations, we systemically study their electronic and magnetic properties, and reveal some important findings to obtain low dimensional magnetic materials with high working temperature. All the 2D $\mathrm{Fe}_{n} \mathrm{GeTe}_{2}$ ultrathin films considered here are robust ferromagnetic/ferrimagnetic with magnetic transition temperature of 68-570 K, which can be ascribed to the coexistence of itinerant and localized electronic state. The localized magnetism comes from the electrons in $d_{\mathrm{z} 2}$ orbital, while the itinerant magnetism derive from electrons in $d_{\mathrm{xz}} / d_{\mathrm{yz}} / d_{\mathrm{xy}} / d_{\mathrm{x} 2-\mathrm{y} 2}$ orbitals. The coexistence of itinerant and localized electronic states also shows a good correlation with many critical magnetic parameters, such as magnetic moment, exchange parameters, $\mathrm{MAE}$ and $\mathrm{T}_{\mathrm{C}}$, which have been carefully discussed in this paper. Based on these results, we propose a localized Fe-Fe exchange Heisenberg model, which can be well described the exchange between the $d_{\mathrm{z} 2}$ orbitals in FGT systems. It may also suitable to the variable valence element-based magnetic compounds. Meanwhile, the itinerant magnetism introduced to our mechanism to explain the competitive intra- and interlayer ferromagnetism. Moreover, the established thickness-dependent magnetic order suggests the possibility of tuning the interlayer exchange energy of Fe-Ge-Te system by changing the composition. Our studies are very helpful to understand the modulating effect of thickness on $2 \mathrm{D} \mathrm{Fe}_{n} \mathrm{GeTe}_{2}$ ultrathin films 
with variable valence elements and indicate that $2 \mathrm{D}$ magnetic $\mathrm{Fe}_{n} \mathrm{GeTe}_{2}$ ultrathin films can be a very promising candidate for future room temperature spintronic applications.

\section{Methods}

First-principle calculations. Our first-principles calculations were based on density functional theory (DFT) within the generalized gradient approximation (GGA), ${ }^{53}$ as implemented in the VASP code. ${ }^{54}$ The projected augmented wave (PAW) potentials was used to describe the ion-electron interaction. ${ }^{55}$ The energy cutoff of plane wave basis was set as 500 eV. A vacuum space of $20 \AA$ thickness was added to avoid the interaction between adjacent layers. During geometry optimization, a Monkhorst-Pack k-point mesh of $0.02 \AA^{-1}$ were chosen for sampling the 2D Brillouin zones. To remove the self-interaction errors, the effective Hubbard $U$ parameter $(U=4.3 \mathrm{eV})$ was included within the $\mathrm{PBE}+\mathrm{U}$ framework, which is consistent with previous studies. ${ }^{56,57}$ Correction of van der Waals interactions using the DFTD3 scheme, ${ }^{58}$ was included in the bulk $\mathrm{Fe}_{3} \mathrm{GeTe}_{2}$ calculations.

To describe the magnetic properties of $\mathrm{Fe}_{\mathrm{n}} \mathrm{GeTe}_{2}$ crystals, the magnetic anisotropy energy (MAE) is defined as

$$
\mathrm{MAE}=E_{\mathrm{tot}}[100]-E_{\mathrm{tot}}[001]
$$

where $E_{\text {tot }}[100]$ and $E_{\text {tot }}[001]$ refer to the total energies of states whose magnetization direction is parallel and perpendicular to the basal plane, respectively. ${ }^{59}$

In the present system, the minority spin states dominate the magnetic anisotropy, such that the MAE can be expressed as ${ }^{60}$

$$
\mathrm{MAE} \approx \Delta \mathrm{E}^{\mathrm{dd}}=(\xi)^{2} \sum_{\mathrm{o}^{-} \mathrm{u}^{-}} \frac{\left|\left\langle o^{-}\left|L_{z}\right| u^{-}\right\rangle\right|^{2}-\left|\left\langle o^{-}\left|L_{x}\right| u^{-}\right\rangle\right|^{2}}{\varepsilon_{\mathrm{u}^{-}-\varepsilon_{0^{-}}}}
$$

Here, $L_{x}$ and $L_{z}$ are the $x$ and $z$ components of angular momentum operator, and o and $\mathrm{u}$ denote the spin-down orbital in the occupied and unoccupied states, respectively. From Eq.(2), we know that $\Delta \mathrm{E}^{\mathrm{dd}}$ is not only determined by the orbital character of the occupied states but also depends on the coupling with the empty states and splitting between them through the energy denominator.

Moreover, Monte Carlo (MC) simulations have been carried out to determine the magnetic transition temperatures. The Hamiltonian of the system is expressed as following form 


$$
\left.H=-\sum_{(i j)} J_{i j}^{\text {UI }} S_{i} \cdot S_{j}^{\text {UI }}-K \sum_{i} \underset{S_{i}}{\stackrel{\text { III }}{\mathrm{r}} \cdot e_{i}}\right)^{2}
$$

where $\stackrel{\text { ". }}{S_{i}}$ is the unit vector of the magnetic moment at the site $i ; J_{i j}$ represents the exchangecoupling constant between magnetic Fe atoms; $K$ is the anisotropy constant and ${ }_{e} e_{i}$ is the unit vector along the easy direction of the magnetic anisotropy. The parameters used in the $\mathrm{MC}$ simulations were obtained from first-principles calculations. To determine the Curie temperature, the magnetization $M$ per atom and the specific heat $C_{\mathrm{m}}$ are calculated by

$$
M=\left\langle\left[\left(\sum_{i} S_{i}^{x}\right)^{2}+\left(\sum_{i} S_{i}^{y}\right)^{2}+\left(\sum_{i} S_{i}^{z}\right)^{2}\right]^{1 / 2}\right\rangle / N
$$

and

$$
\mathrm{C}_{m}=\left(\left\langle E^{2}\right\rangle-\langle E\rangle^{2}\right) / N k_{B} T^{2}
$$

respectively. Here $N$ is the total number of magnetic Fe atoms, and $k_{\mathrm{B}}$ denotes the Boltzmann constant. The simulation supercells were constructed by $50 \times 50$ expansion of the unit cell. For each temperature, the first $10^{5} \mathrm{MC}$ steps were discarded for thermal equilibration; the successive $10^{5} \mathrm{MC}$ steps were then used to collect data and determine the thermodynamic averages of given physical quantities. Indeed, all thermodynamic properties were averaged over five different seed numbers.

\section{Data availability}

The data that support the findings of this study are available from the corresponding author on reasonable request.

\section{References}

1. Huang, B. et al. Layer-dependent ferromagnetism in a van der Waals crystal down to the monolayer limit. Nature 546, 270-273 (2017).

2. McGuire, M. Crystal and Magnetic Structures in Layered, Transition Metal Dihalides and Trihalides. Crystals 7, 121 (2017).

3. Li, Z., Zhou, B. \& Luan, C. Strain-tunable magnetic anisotropy in two-dimensional Dirac halfmetals: nickel trihalides. RSC Adv. 9, 35614-35623 (2019).

4. Gong, C. et al. Discovery of intrinsic ferromagnetism in two-dimensional van der Waals crystals. Nature 546, 265-269 (2017).

5. Vatansever, E. et al. Strain effects on electronic and magnetic properties of the monolayer $\alpha-$ $\mathrm{RuCl}_{3}$ : A first-principles and Monte Carlo study. J. Appl. Phys. 125, 083903 (2019).

6. Ersan, F. et al. Exploring the electronic and magnetic properties of new metal halides from bulk 
to two-dimensional monolayer: $\mathrm{RuX}_{3}(\mathrm{X}=\mathrm{Br}, \mathrm{I})$. J. Magn. Magn. Mater. 476, 111-119 (2019).

7. Goodenough, J. B. Theory of the Role of Covalence in the Perovskite-Type Manganites [La, M(II)] $\mathrm{MnO}_{3}$. Phys. Rev. 100, 564-573 (1955).

8. Kanamori, J. Crystal Distortion in Magnetic Compounds. J. Appl. Phys. 31, S14-S23 (1960).

9. Anderson, P. W. New Approach to the Theory of Superexchange Interactions. Phys. Rev. 115, 2-13 (1959).

10. Bhatti, S., Sbiaa, R., Hirohata, A., Ohno, H., Fukami, S. \& Piramanayagam, S. N. Spintronics based random access memory: a review. Mater. Today 20, 530-548 (2017).

11. Zhang, Y. et al. Ultrathin Magnetic 2D Single-Crystal CrSe. Adv. Mater. 31, 1900056 (2019).

12. Kang, L. et al. Phase-controllable growth of ultrathin 2D magnetic FeTe crystals. Nat. Commun. 11, 3729 (2020).

13. Fei, Z. et al. Two-dimensional itinerant ferromagnetism in atomically thin $\mathrm{Fe}_{3} \mathrm{GeTe}_{2}$. Nat. Mater. 17, 778-782 (2018).

14. Deng, Y. et al. Gate-tunable room-temperature ferromagnetism in two-dimensional $\mathrm{Fe}_{3} \mathrm{GeTe}_{2}$. Nature 563, 94-99 (2018).

15. Seo, J. et al. Nearly room temperature ferromagnetism in a magnetic metal-rich van der Waals metal. Sci. Adv. 6, eaay8912 (2020).

16. May, A. F. et al. Ferromagnetism Near Room Temperature in the Cleavable van der Waals Crystal $\mathrm{Fe}_{5} \mathrm{GeTe}_{2}$. ACS Nano 13, 4436-4442 (2019).

17. Yuan, Q.-Q. et al. Ferromagnetic MnSn Monolayer Epitaxially Grown on Silicon Substrate. Chinese Phys. Lett. 37, 077502 (2020).

18. Abdullahi, Y. Z., Vatansever, Z. D., Ersan, F., Akinci, U., Akturk, O. U. \& Akturk, E. Ferromagnetic $\mathrm{TM}_{2} \mathrm{BC}(\mathrm{TM}=\mathrm{Cr}, \mathrm{Mn})$ monolayers for spintronic devices with high Curie temperature. Phys. Chem. Chem. Phys. 23, 6107-6115 (2021).

19. Gökoğlu, G. \& Aktürk, E. Half metallicity and pressure-induced electronic structure of monolayer $\mathrm{FeX}_{2}(\mathrm{X}=\mathrm{S}, \mathrm{Se})$. Mater. Res. Express 4, 116305 (2017).

20. Stoner, E. C. Collective electron ferronmagnetism. Proceedings of the Royal Society of London. Series A. Mathematical and Physical Sciences 165, 372-414 (1938).

21. Wang, H. et al. Pressure-Dependent Intermediate Magnetic Phase in Thin $\mathrm{Fe}_{3} \mathrm{GeTe}_{2}$ Flakes. $J$. Phys. Chem. Lett. 11, 7313-7319 (2020).

22. $\mathrm{Xu}, \mathrm{X}$. et al. Signature for non-Stoner ferromagnetism in the van der Waals ferromagnet $\mathrm{Fe}_{3} \mathrm{GeTe}_{2}$. Phys. Rev. B 101, 201104 (2020).

23. Henkelman, G., Arnaldsson, A. \& Jónsson, H. A fast and robust algorithm for Bader decomposition of charge density. Comp. Mater. Sci. 36, 354-360 (2006).

24. Deiseroth, H.-J., Aleksandrov, K., Reiner, C., Kienle, L. \& Kremer, R. K. $\mathrm{Fe}_{3} \mathrm{GeTe}_{2}$ and $\mathrm{Ni}_{3} \mathrm{GeTe}_{2}$ - Two New Layered Transition-Metal Compounds: Crystal Structures, HRTEM Investigations, and Magnetic and Electrical Properties. Eur. J. Inorg. Chem. 2006, 1561-1567 (2006).

25. Kou, S.-P., Li, T. \& Weng, Z.-Y. Coexistence of itinerant electrons and local moments in ironbased superconductors. Europhys. Lett. 88, 17010 (2009).

26. Tovar, M. et al. Evidence of strong antiferromagnetic coupling between localized and itinerant electrons in ferromagnetic $\mathrm{Sr}_{2} \mathrm{FeMoO}_{6}$. Phys. Rev. B 66, 024409 (2002).

27. Edwards, D. M. Ferromagnetism and electron-phonon coupling in the manganites. Adv. Phys. 51, 1259-1318 (2002). 
28. Bhide, V. G., Rajoria, D. S., Rao, C. N. R., Rao, G. R. \& Jadhao, V. G. Itinerant-electron ferromagnetism in $\mathrm{La}_{1-\mathrm{x}} \mathrm{Sr}_{\mathrm{x}} \mathrm{CoO}_{3}$ : A Mössbauer study. Phys. Rev. B 12, 2832-2843 (1975).

29. Stöhr, J. \& Siegmann, H. C. Magnetism (Springer-Verlag, Berlin, Germany, 2006).

30. Moritomo, Y., Asamitsu, A. \& Tokura, Y. Pressure effect on the double-exchange ferromagnet $\mathrm{La}_{1-\mathrm{x}} \mathrm{Sr}_{\mathrm{x}} \mathrm{MnO}_{3}(0.15<=\mathrm{x}<=0.5)$. Phys. Rev. B 51, 16491-16494 (1995).

31. Yi, J. et al. Competing antiferromagnetism in a quasi-2D itinerant ferromagnet: $\mathrm{Fe}_{3} \mathrm{GeTe}_{2} .2 D$ Materials 4, 011005 (2016).

32. Fu, Z., Chen, H., Liu, Y., Liu, M. \& Liu, W.-M. Interface-induced ferroelectric domains and charged domain walls in $\mathrm{BiFeO}_{3} / \mathrm{SrTiO}_{3}$ superlattices. Phys. Rev. B 103, 195301 (2021).

33. Hu, X., Zhao, Y., Shen, X., Krasheninnikov, A. V., Chen, Z. \& Sun, L. Enhanced Ferromagnetism and Tunable Magnetism in $\mathrm{Fe}_{3} \mathrm{GeTe}_{2}$ Monolayer by Strain Engineering. ACS Appl. Mater. Inter. 12, 26367-26373 (2020).

34. Shabbir, B. et al. Long range intrinsic ferromagnetism in two dimensional materials and dissipationless future technologies. Appl. Phys. Rev. 5, 041105 (2018).

35. Wang, P., Jiang, X., Hu, J. \& Zhao, J. Chemically Engineering Magnetic Anisotropy of 2D Metalloporphyrin. Adv. Sci. 4, 1700019 (2017).

36. Park, S. Y. et al. Controlling the Magnetic Anisotropy of the van der Waals Ferromagnet $\mathrm{Fe}_{3} \mathrm{GeTe}_{2}$ through Hole Doping. Nano Lett. 20, 95-100 (2020).

37. Kim, D. et al. Antiferromagnetic coupling of van der Waals ferromagnetic $\mathrm{Fe}_{3} \mathrm{GeTe}_{2}$. Nanotechnology 30, 245701 (2019).

38. May, A. F., Calder, S., Cantoni, C., Cao, H. \& McGuire, M. A. Magnetic structure and phase stability of the van der Waals bonded ferromagnet $\mathrm{Fe}_{3-\mathrm{x}} \mathrm{GeTe}_{2}$. Phys. Rev. B 93, 014411 (2016).

39. Villars, P., Calvert, L. D. \& Pearson, W. B. Handbook of Crystallographic Data for Intermetallic Phases. Acta Crystallogr. A 40, C444 (1984).

40. Behler, J. Atom-centered symmetry functions for constructing high-dimensional neural network potentials. J. Chem. Phys. 134, 074106 (2011).

41. Chu, J. et al. Sub-millimeter-Scale Growth of One-Unit-Cell-Thick Ferrimagnetic $\mathrm{Cr}_{2} \mathrm{~S}_{3}$ Nanosheets. Nano Lett. 19, 2154-2161 (2019).

42. Néel, M. L. Propriétés magnétiques des ferrites ; ferrimagnétisme et antiferromagnétisme. Ann. Phys. 12, 137-198 (1948).

43. Stinshoff, R. et al. Completely compensated ferrimagnetism and sublattice spin crossing in the half-metallic Heusler compound $\mathrm{Mn}_{1.5} \mathrm{FeV}_{0.5} \mathrm{Al}$. Phys. Rev. B 95, 060410 (2017).

44. Midhunlal, P. V., Arout Chelvane, J., Prabhu, D., Gopalan, R. \& Harish Kumar, N. $\mathrm{Mn}_{2} \mathrm{~V}_{0.5} \mathrm{Co}_{0.5} \mathrm{Z}(\mathrm{Z}=\mathrm{Ga}, \mathrm{Al})$ Heusler alloys: High $\mathrm{T}_{\mathrm{C}}$ compensated P-type ferrimagnetism in arc melted bulk and N-type ferrimagnetism in melt-spun ribbons. J. Magn. Magn. Mater. 489, 165298 (2019).

45. Bitla, Y. et al. Origin of metallic behavior in $\mathrm{NiCo}_{2} \mathrm{O}_{4}$ ferrimagnet. Sci. Rep-UK 5, 15201 (2015).

46. Nuttall, C. J. \& Day, P. Magnetization of the Layer Compounds $A F e^{\mathrm{II}} \mathrm{Fe}^{\mathrm{III}}\left(\mathrm{C}_{2} \mathrm{O}_{4}\right)_{3}(A=$ Organic Cation), in Low and High Magnetic Fields: Manifestation of Néel $\mathrm{N}$ and $\mathrm{Q}$ Type Ferrimagnetism in a Molecular Lattice. Chem. Mater. 10, 3050-3057 (1998).

47. Vatansever, E. \& Polat, H. Monte Carlo investigation of a spherical ferrimagnetic core-shell nanoparticle under a time dependent magnetic field. J. Magn. Magn. Mater. 343, 221-227 (2013).

48. Mahat, R. et al. Tuneable structure and magnetic properties in $\mathrm{Fe}_{3-\mathrm{x}} \mathrm{V}_{\mathrm{x}}$ Ge alloys. J. Alloys Compd. 830, 154403 (2020). 
49. Mahat, R. et al. Influence of Cr-substitution on the structural, magnetic, electron transport, and mechanical properties of $\mathrm{Fe}_{3-\mathrm{x}} \mathrm{Cr}_{\mathrm{x}} \mathrm{Ge}$ Heusler alloys. J. Magn. Magn. Mater. 521, 167398 (2021).

50. $\mathrm{Xu}, \mathrm{L}$. et al. Magnetic entropy change and accurate determination of Curie temperature in single-crystalline helimagnet FeGe. Europhys. Lett. 117, 47004 (2017).

51. Yasukōchi, K., Kanematsu, K. \& Ohoyama, T. Magnetic Properties of Intermetallic Compounds in Iron-Germanium System : $\mathrm{Fe}_{1.67} \mathrm{Ge}$ and $\mathrm{FeGe}_{2}$. J. Phys. Soc. Jpn. 16, 429-433 (1961).

52. Fukuma, Y., Asada, H., Miyashita, J., Nishimura, N. \& Koyanagi, T. Magnetic properties of IVVI compound GeTe based diluted magnetic semiconductors. J. Appl. Phys. 93, 7667-7669 (2003).

53. Perdew, J. P., Burke, K. \& Ernzerhof, M. Generalized Gradient Approximation Made Simple. Phys. Rev. Lett. 77, 3865-3868 (1996).

54. Hafner, J. Ab-initio simulations of materials using VASP: Density-functional theory and beyond. J. Comput. Chem. 29, 2044-2078 (2008).

55. Blochl, P. E. Projector augmented-wave method. Phys. Rev. B 50, 17953-17979 (1994).

56. Mosey, N. J., Liao, P. \& Carter, E. A. Rotationally invariant ab initio evaluation of Coulomb and exchange parameters for DFT+U calculations. J. Chem. Phys. 129, 014103 (2008).

57. Zhuang, H. L., Kent, P. R. C. \& Hennig, R. G. Strong anisotropy and magnetostriction in the two-dimensional Stoner ferromagnet $\mathrm{Fe}_{3} \mathrm{GeTe}_{2}$. Phys. Rev. B 93, 134407 (2016).

58. Grimme, S., Antony, J., Ehrlich, S. \& Krieg, H. A consistent and accurate ab initio parametrization of density functional dispersion correction (DFT-D) for the 94 elements H-Pu. J. Chem. Phys. 132, 154104 (2010).

59. Abdullahi, Y. Z., Ersan, F., Vatansever, Z. D., Aktürk, E. \& Aktürk, O. Ü. Exploring the potential of $\mathrm{MnX}(\mathrm{S}, \mathrm{Sb})$ monolayers for antiferromagnetic spintronics: A theoretical investigation. $J$. Appl. Phys. 128, 113903 (2020).

60. Xing, J., Wang, P., Jiang, Z., Jiang, X., Wang, Y. \& Zhao, J. Rational design of 2D organic magnets with giant magnetic anisotropy based on two-coordinate $5 \mathrm{~d}$ transition metals. $A P L$ Mater. 8, 071105 (2020).

\section{Acknowledgement}

This work was supported by the National Natural Science Foundation of China (11874097, 91961204, 11804044) and the Fundamental Research Funds for the Central Universities of China (DUT19LK12). We acknowledge the project of Dalian Youth Science and Technology Star (2017RQ012). The authors also acknowledge the Supercomputing Center of Dalian University of Technology for providing the computing resources.

\section{Author contributions}

X.J. supervised this study. X.J., Y.Q. and J.J.Z. conceived the idea. Q.X.L. performed the theoretical simulations. J.P.X., Z.J. and Y.G. participated in the discussion of results. X.J. and Q.X.L. drafted the manuscript. J.J.Z. edited the manuscript. All the authors contributed to the overall scientific interpretation. 


\section{Competing interests}

The authors declare no competing interests.

\section{Additional information}

Supplementary Information accompanies this paper.

Correpondence and request for material should addressed to X.J. or Y.Q.

Reprint and permission information is available online at http://www.nature.com/reprints

Publisher's note Springer Nature remains neutral with regard to jurisdictional claim in piblished maps and institutional affiliations. 


\section{Supplementary Files}

This is a list of supplementary files associated with this preprint. Click to download.

- SupportingInformation.docx 